\title{
RATIO PROPERTIES IN LOCALLY COMPACT AMENABLE GROUPS
}

\author{
BY \\ WILLIAM R. EMERSON
}

0 . Introduction and summary. Let $\Gamma$ be a locally compact (Hausdorff) topological group, and let $|*|$ denote a fixed left Haar measure on the Borel sets of $\Gamma$ (in the sense of [1, §51]). It is known (see [2]) that the existence of a left invariant mean on $L^{\infty}(\Gamma)$ is equivalent to the Følner Condition (FC):

To any $\varepsilon>0$ and any compact set $C \subseteq \Gamma$, there corresponds a Borel set $V$ of finite nonzero measure such that

$$
|\gamma V \triangle V| /|V|<\varepsilon
$$

for all $\gamma \in C$.

The purpose of the present paper is the investigation of sequences $\left\{V_{n}\right\}$ of Borel sets of $\Gamma$ which eventually satisfy either condition (FC) for every $\varepsilon>0$ and compact $C \subseteq \Gamma$, or a stronger condition. More precisely, we say a sequence $\left\{V_{n}\right\}$ of Borel sets of $\Gamma$ has the weak ratio property, written $\left\{V_{n}\right\} \in W_{\Gamma}$, if and only if for all $\gamma \in \Gamma$

$$
\operatorname{Lim}_{n \rightarrow \infty} \frac{\left|\gamma V_{n} \triangle V_{n}\right|}{\left|V_{n}\right|}=0
$$

Similarly $\left\{V_{n}\right\}$ is said to have the strong ratio property, written $\left\{V_{n}\right\} \in S_{\Gamma}$, if and only if for all compact $C \neq \varnothing$ in $\Gamma$,

$$
\operatorname{Lim}_{n \rightarrow \infty} \frac{\left|C V_{n} \triangle V_{n}\right|}{\left|V_{n}\right|}=0
$$

In $\S \mathrm{I}$ we consider primarily properties of individual sequences of sets with ratio properties, and obtain particularly detailed results in case $\Gamma$ is Abelian. We also show that $W_{\Gamma} \neq \varnothing$ if and only if $\Gamma$ is amenable and $\sigma$-compact. In $\S$ II properties of the sets $W_{\Gamma}$ and $S_{\Gamma}$ are investigated, and we show that $S_{\Gamma}$ is always "dense" in $W_{\Gamma}$. From this result we conclude that $S_{\Gamma} \neq \varnothing$ if and only if $\Gamma$ is amenable and $\sigma$-compact, and obtain necessary conditions for a sequence of sets to have the weak ratio property. Finally, in §III a generalization of the weak ratio property is made to product measure spaces $\Gamma \times S$, where $S$ is any positive measure space, and the case of finitely generated discrete Abelian $\Gamma$ is considered in detail.

The following notational conventions are observed throughout: $N$ is the set of nonnegative integers; $N^{+}$is the set of positive integers. If $\Gamma$ is a topological group,

Received by the editors April 10, 1967. 
$e$ denotes the unit of $\Gamma, \bar{V}$ denotes the closure of $V \subseteq \Gamma, \Delta(\gamma)$ denotes the modular function of $\Gamma$ evaluated at $\gamma \in \Gamma$, and we frequently use additive notation in case $\Gamma$ is Abelian. Finally, $A \sim B$ denotes the difference of the sets $A$ and $B$ and $I_{V}$ denotes the characteristic function of the set $V$.

\section{Sequences of sets satisfying the ratio properties.}

I.1. Definition of the ratio properties and equivalent formulations. We first make the following basic definitions:

Definition 1. A sequence $\left\{V_{n}\right\}$ of Borel sets of $\Gamma$ of finite nonzero measure is said to satisfy the weak ratio property with respect to $\Gamma$ if and only if

$$
\operatorname{Lim}_{n \rightarrow \infty} \frac{\left|\gamma V_{n} \triangle V_{n}\right|}{\left|V_{n}\right|}=0
$$

for all $\gamma \in \Gamma$. Denote the collection of all such sequences by $W_{\Gamma}$. Furthermore, let $W_{\Gamma}^{0}$ be the subset of $W_{\Gamma}$ consisting of those sequences $\left\{V_{n}\right\}$ with only finitely many $\bar{V}_{n}$ noncompact.

Definition 2. A sequence $\left\{V_{n}\right\}$ of Borel sets of $\Gamma$ of finite nonzero measure is said to satisfy the strong ratio property with respect to $\Gamma$ if and only if

$$
\operatorname{Lim}_{n \rightarrow \infty} \frac{\left|C V_{n} \triangle V_{n}\right|}{\left|V_{n}\right|}=0
$$

for all compact subsets $C \neq \varnothing \varnothing$ of $\Gamma$. Denote the collection of all such sequences by $S_{\Gamma}$. Furthermore, let $S_{\Gamma}^{0}$ be the subset of $S_{\Gamma}$ consisting of those sequences $\left\{V_{n}\right\}$ with only finitely many $\bar{V}_{n}$ noncompact.

The following theorems give some conditions equivalent to the ratio properties:

THEOREM 1. If $\left\{V_{n}\right\}$ is a sequence of Borel sets of $\Gamma$ with finite nonzero measure, the following are equivalent:

(i) $\left\{V_{n}\right\} \in W_{\Gamma}$.

(ii) $\operatorname{Lim}_{n \rightarrow \infty}\left(\left|C V_{n}\right| /\left|V_{n}\right|\right)=1$ for all finite subsets $C \neq \varnothing$ of $\Gamma$.

(iii) $\operatorname{Lim}_{n \rightarrow \infty}\left(\left|\bigcap_{\gamma \in C} \gamma V_{n}\right| /\left|V_{n}\right|\right)=1$ for all finite subsets $C \neq \varnothing$ of $\Gamma$.

Proof. (ii) implies (i) upon setting $C=\{\gamma, e\}$ and noting that $C V_{n}=\gamma V_{n} \cup V_{n}$ and $\gamma V_{n} \triangle V_{n} \subseteq\left(C V_{n} \sim V_{n}\right) \cup\left(C V_{n} \sim \gamma V_{n}\right)$ and therefore

$$
\begin{aligned}
\operatorname{Limsup}_{n \rightarrow \infty} \frac{\left|\gamma V_{n} \triangle V_{n}\right|}{\left|V_{n}\right|} & \leqq \operatorname{Limsup}_{n \rightarrow \infty} \frac{\left(\left|C V_{n} \sim V_{n}\right|+\left|C V_{n} \sim \gamma V_{n}\right|\right)}{\left|V_{n}\right|} \\
& =\operatorname{Limsup}_{n \rightarrow \infty} \frac{2\left(\left|C V_{n}\right|-\left|V_{n}\right|\right)}{\left|V_{n}\right|}=0 .
\end{aligned}
$$

(iii) also implies (i) upon setting $C=\{\gamma, e\}$ and noting that

$$
\gamma V_{n} \triangle V_{n} \subseteq\left(\gamma V_{n} \sim\left(\gamma V_{n} \cap V_{n}\right)\right) \cup\left(V_{n} \sim\left(\gamma V_{n} \cap V_{n}\right)\right)
$$

and therefore

$$
\underset{n \rightarrow \infty}{\operatorname{Limsup}} \frac{\left|\gamma V_{n} \triangle V_{n}\right|}{\left|V_{n}\right|} \leqq \operatorname{Limsup}_{n \rightarrow \infty} \frac{\left(\left|\gamma V_{n}\right|-\left|\gamma V_{n} \cap V_{n}\right|+\left|V_{n}\right|-\left|\gamma V_{n} \cap V_{n}\right|\right)}{\left|V_{n}\right|}=0 .
$$


Now to show (i) implies (ii), let $C=\left\{\gamma_{1}, \ldots, \gamma_{x}\right\}$ be any nonempty finite subset of $\Gamma$. Therefore $\left\{V_{n}\right\} \in W_{\Gamma}$ implies

$$
\operatorname{Lim}_{n \rightarrow \infty} \frac{\left|\gamma_{i} V_{n} \triangle V_{n}\right|}{\left|V_{n}\right|}=0 \text { for } 1 \leqq i \leqq \kappa .
$$

But $\gamma_{i} V_{n} \subseteq\left(\gamma_{i} V_{n} \triangle V_{n}\right) \cup V_{n}$, and therefore

$$
C V_{n}=\bigcup_{1 \leqq 1 \leq \kappa} \gamma_{i} V_{n} \subseteq \bigcup_{1 \leqq 1 \leq \kappa}\left(\gamma_{i} V_{n} \triangle V_{n}\right) \cup V_{n}
$$

which implies

$$
1 \leqq \operatorname{Limsup}_{n \rightarrow \infty} \frac{\left|C V_{n}\right|}{\left|V_{n}\right|} \leqq \operatorname{Limsup}_{n \rightarrow \infty} \frac{\sum_{1 \leq i \leq x}\left|\gamma_{i} V_{n} \Delta V_{n}\right|+\left|V_{n}\right|}{\left|V_{n}\right|}=1 .
$$

Finally to show that (i) implies (iii) we note that

$$
\bigcap_{\gamma \in C} \gamma V_{n}=\left\{\left\{\cdots\left\{\left(\gamma_{1} V_{n}\right) \sim\left(\gamma_{1} V_{n} \triangle \gamma_{2} V_{n}\right)\right\} \sim \cdots\right\} \sim\left(\gamma_{x-1} V_{n} \triangle \gamma_{x} V_{n}\right)\right\}
$$

and consequently

$$
\begin{aligned}
1 & \geqq \operatorname{Liminf}_{n \rightarrow \infty} \frac{\left|\bigcap_{\gamma \in C} \gamma V_{n}\right|}{\left|V_{n}\right|} \geqq \operatorname{Liminf}_{n \rightarrow \infty} \frac{\left|V_{n}\right|-\sum_{1 \leqq i<x}\left|\gamma_{i} V_{n} \Delta \gamma_{i+1} V_{n}\right|}{\left|V_{n}\right|} \\
& =1-\operatorname{Limsup}_{n \rightarrow \infty} \sum_{1 \leqq i<x} \frac{\left|\gamma_{i+1}^{-1} \gamma_{i} V_{n} \Delta V_{n}\right|}{\left|V_{n}\right|}=1 .
\end{aligned}
$$

THEOREM 2. If $\left\{V_{n}\right\}$ is a sequence of Borel sets of $\Gamma$ with finite nonzero measure, $\left\{V_{n}\right\} \in S_{\Gamma}$ if and only if $\operatorname{Lim}_{n \rightarrow \infty}\left|C V_{n}\right| /\left|V_{n}\right|=1$ for all compact subsets. $C \neq \varnothing$ of $\Gamma$.

Proof. Let $\left\{V_{n}\right\} \in S_{\Gamma}$, and let $C \neq \varnothing$ be any compact subset of $\Gamma$. Then

$$
0 \leqq \operatorname{Limsup}_{n \rightarrow \infty} \frac{\left|C V_{n}\right|-\left|V_{n}\right|}{\left|V_{n}\right|} \leqq \operatorname{Limsup}_{n \rightarrow \infty} \frac{\left|C V_{n} \sim V_{n}\right|}{\left|V_{n}\right|} \leqq \operatorname{Limsup}_{n \rightarrow \infty} \frac{\left|C V_{n} \triangle V_{n}\right|}{\left|V_{n}\right|}=0,
$$

and consequently $\operatorname{Lim}_{n \rightarrow \infty}\left|C V_{n}\right| /\left|V_{n}\right|=1$.

Conversely if $C \neq \varnothing$ is any compact subset of $\Gamma$, let $C^{\prime}=C \cup\{e\}$ and therefore $\operatorname{Lim}_{n \rightarrow \infty}\left|C^{\prime} V_{n}\right| /\left|V_{n}\right|=1$. But $C^{\prime} V_{n}=V_{n} \cup C V_{n}=V_{n} \cup\left(C V_{n} \sim V_{n}\right)=$ $C V_{n} \cup\left(V_{n} \sim C V_{n}\right)$ and therefore, since $\left|C V_{n}\right| \geqq\left|V_{n}\right|, \operatorname{Lim}_{n \rightarrow \infty}\left|C V_{n} \sim V_{n}\right| /\left|V_{n}\right|=$ $\operatorname{Lim}_{n \rightarrow \infty}\left|V_{n} \sim C V_{n}\right| /\left|V_{n}\right|=0$ implying $\operatorname{Lim}_{n \rightarrow \infty}\left|C V_{n} \triangle V_{n}\right| /\left|V_{n}\right|=0$.

I.2. Uniformity of convergence. We now show that the pointwise convergence to 0 in the definition of $W_{\Gamma}$ is equivalent to the formally stronger condition of uniform convergence to 0 on compacta, and derive some useful corollaries. The following two lemmas are pivotal in the proof of Theorem 3:

LemMA 1. Let $K$ be a compact neighborhood of the unit in $\Gamma$, and let $\left\{f_{n}\right\}$ be a sequence of uniformly bounded positive measurable functions on $\Gamma$ such that

(1) $\operatorname{Lim}_{n \rightarrow \infty} f_{n}(\gamma)=0$ for all $\gamma \in K^{2}$, 
(2) There exists an absolute constant $c>0$ such that $f_{n}(\alpha \beta) \geqq c\left|f_{n}(\alpha)-f_{n}(\beta)\right|$ for all $\alpha, \beta \in K$.

Then $f_{n} \rightarrow 0$ uniformly on $K$.

Proof. Let $S_{n}(\varepsilon)=\left\{\gamma \in K^{2}: f_{n}(\gamma) \geqq \varepsilon\right\}$ for $n \in N^{+}$and $\varepsilon>0$. Since pointwise convergence implies convergence in measure $\left(\left|K^{2}\right|<+\infty\right)$ we conclude $\operatorname{Lim}_{n \rightarrow \infty}\left|S_{n}(\varepsilon)\right|=0$ for all $\varepsilon>0$. Now assume convergence to 0 on $K$ is not uniform. Then without loss of generality (extracting a subsequence if necessary) we may assume that there exists an $\eta>0$ and a sequence $\left\{\alpha_{n}\right\} \subseteq K$ such that $f_{n}\left(\alpha_{n}\right)>\eta$. Let $T_{n}(\varepsilon)=S_{n}(\varepsilon) \cap K$, and consider $B_{n}=T_{n}(\eta / 2) \cup \alpha_{n}\left(K \sim T_{n}(\eta / 2)\right) \subseteq K^{2}$. First $\left|B_{n}\right|$ $\geqq|K| / 2>0$ by left invariance of Haar measure $(|K|>0$ since $K$ has nonempty interior). But also $B_{n} \subseteq S_{n}\left(\varepsilon_{0}\right)$, where $\varepsilon_{0}=\operatorname{Min}\{\eta / 2, c \eta / 2\}$. For $\gamma \in B_{n}$ implies either $f_{n}(\gamma) \geqq \eta / 2$ in case $\gamma \in T_{n}(\eta / 2)$, or else $\gamma=\alpha_{n} \beta$ where $\beta \in K \sim T_{n}(\eta / 2)$ and hence by (2) $f_{n}(\gamma)=f_{n}\left(\alpha_{n} \beta\right) \geqq c\left|f_{n}\left(\alpha_{n}\right)-f_{n}(\beta)\right| \geqq c|\eta-\eta / 2|=c \eta / 2$. But this clearly violates $\operatorname{Lim}_{n \rightarrow \infty}\left|S_{n}\left(\varepsilon_{0}\right)\right|=0$.

LEMMA 2. Let $X$ be a Borel set of finite nonzero measure in the locally compact group $\Gamma$, and let $f_{X}(\gamma)=|\gamma X \triangle X| /|X|$ for $\gamma \in \Gamma$. Then for all $\alpha, \beta \in \Gamma, f_{X}(\alpha \beta) \geqq$ $\left|f_{X}(\alpha)-f_{X}(\beta)\right|$.

Proof. We apply the metric property of symmetric difference and the left invariance of Haar measure to obtain

$$
\begin{aligned}
& |\beta X \triangle X| \leqq\left|\beta X \triangle \alpha^{-1} X\right|+\left|\alpha^{-1} X \triangle X\right|=|\alpha \beta X \triangle X|+|X \triangle \alpha X|, \\
& |\alpha X \triangle X| \leqq|\alpha X \triangle \alpha \beta X|+|\alpha \beta X \triangle X|=|X \triangle \beta X|+|\alpha \beta X \triangle X| .
\end{aligned}
$$

Upon combining these two inequalities we obtain the assertion.

We now prove the "uniform" analogue of Theorem 1:

THEOREM 3. If $\left\{V_{n}\right\}$ is a sequence of Borel sets of $\Gamma$ with finite nonzero measure, the following are equivalent:

(i) $\left\{V_{n}\right\} \in W_{\Gamma}$.

(ii) $\left|\gamma V_{n} \triangle V_{n}\right| /\left|V_{n}\right| \rightarrow 0$ uniformly for $\gamma$ in compacta.

(iii) If $K$ is any positive integer and $C$ any compact subset of $\Gamma$,

$$
\frac{\left|\bigcap_{1 \leq i \leq k} \gamma_{i} V_{n}\right|}{\left|V_{n}\right|} \rightarrow 1 \text { uniformly for }\left\{\gamma_{1}, \ldots, \gamma_{k}\right\} \subseteq C \text {. }
$$

(iv) If $K$ is any positive integer and $C$ any compact subset of $\Gamma$,

$$
\frac{\left|\left\{\gamma_{1}, \ldots, \gamma_{k}\right\} V_{n}\right|}{\left|V_{n}\right|} \rightarrow 1 \text { uniformly for }\left\{\gamma_{1}, \ldots, \gamma_{k}\right\} \subseteq C \text {. }
$$

Proof. Condition (ii), (iii), or (iv) trivially implies (i), in light of Theorem 1. Conversely, we prove (i) $\Rightarrow$ (ii), (ii) $\Rightarrow$ (iii) and (ii) $\Rightarrow$ (iv):

(i) $\Rightarrow$ (ii): Using the notation of $\operatorname{Lemma}_{2, \operatorname{Lim}_{n \rightarrow \infty}} f_{V_{n}}(\gamma)=0$ for all $\gamma \in \Gamma$. Hence (ii) is an immediate consequence of Lemmas 1 and 2 since each $f_{V_{n}}$ is a continuous function with values in $[0,1]$. 
(ii) $\Rightarrow$ (iii). Fix any compact set $C$ and $\varepsilon>0$. By (ii) there exists an $n_{0}$ such that $\left|\gamma V_{n} \triangle V_{n}\right| /\left|V_{n}\right|<\varepsilon$ for all $n \geqq n_{0}$ and $\gamma$ in the compact set $C_{1}=C^{-1}$. C. Hence

$$
\begin{aligned}
\left|\bigcap_{1 \leqq i \leqq k} \gamma_{i} V_{n}\right| & \geqq\left|\gamma_{1} V_{n}\right|-\sum_{1 \leqq i<k}\left|\gamma_{i} V_{n} \triangle \gamma_{i+1} V_{n}\right| \\
& =\left|V_{n}\right|-\sum_{1 \leqq i<k}\left|\gamma_{i+1}^{-1} \gamma_{i} V_{n} \triangle V_{n}\right| \geqq\left|V_{n}\right|-(k-1) \varepsilon
\end{aligned}
$$

since $\gamma_{i+1}^{-1} \gamma_{i} \in C^{-1} . C=C_{1}$ if $\left\{\gamma_{1}, \ldots, \gamma_{k}\right\} \subseteq C$, and (iii) follows.

(ii) $\Rightarrow$ (iv). $\left\{\gamma_{1}, \ldots, \gamma_{k}\right\} V_{n} \subseteq \bigcup_{1 \leqq i \leqq k}\left(\gamma_{i} V_{n} \triangle V_{n}\right) \cup V_{n}$, implying

$$
1 \leqq \frac{\left|\left\{\gamma_{1}, \ldots, \gamma_{k}\right\} V_{n}\right|}{\left|V_{n}\right|} \leqq \sum_{1 \leqq i \leq k} \frac{\left|\gamma_{i} V_{n} \Delta V_{n}\right|}{\left|V_{n}\right|}+1,
$$

and thus (iv) follows readily from (ii).

COROLlaRY 1. If $\left\{V_{n}\right\} \in W_{\Gamma}$, then $\left\{V_{n}\right\}$ eventually satisfies condition (FC) for every $\varepsilon>0$ and compact $C \subseteq \Gamma$.

Proof. Clear from (ii) of Theorem 3.

As an immediate consequence of the uniformity of convergence we obtain the following corollaries:

COROLlary 2. Let $\left\{V_{n}\right\} \in W_{\Gamma}$, let $C^{\prime}$ be a compact subset of $\Gamma$, and let $\left\{\gamma_{n}^{\prime}\right\}$ be any sequence of elements in $C^{\prime}$. Then $\operatorname{Lim}_{n \rightarrow \infty}\left|\gamma_{n}^{\prime} V_{n} \triangle V_{n}\right| /\left|V_{n}\right|=0$.

Proof. Theorem 3, (ii).

COROLlary 3. Let $\left\{V_{n}\right\} \in W_{\Gamma}$, let $C$ be a compact subset of $\Gamma$, and let $\left\{\gamma_{n}\right\}$ be any sequence of elements in $C$. Then $\left\{\gamma_{n} V_{n}\right\} \in W_{\Gamma}$.

Proof. For any $\gamma \in \Gamma$, upon setting $\gamma_{n}^{\prime}=\gamma_{n}^{-1} \gamma \gamma_{n}$ we obtain

$$
\frac{\left|\gamma \gamma_{n} V_{n} \triangle \gamma_{n} V_{n}\right|}{\left|\gamma_{n} V_{n}\right|}=\frac{\left|\gamma_{n}^{-1} \gamma \gamma_{n} V_{n} \triangle V_{n}\right|}{\left|V_{n}\right|}=\frac{\left|\gamma_{n}^{\prime} V_{n} \triangle V_{n}\right|}{\left|V_{n}\right|},
$$

and upon noting that $\gamma_{n}^{\prime}$ is in the compact set $C^{\prime \prime}=C^{-1} \gamma C$ for all $n \in N^{+}$we immediately obtain Corollary 3 from Corollary 2 .

Remark. Corollary 2 will be essential in the proof of Theorem 13.

I.3. $\Gamma$ for which $W_{\Gamma} \neq \varnothing$. We are now in a position to prove:

THEOREM 4. $W_{\Gamma} \neq \varnothing$ if and only if $\Gamma$ is amenable and $\sigma$-compact.

Proof. Assume $W_{\Gamma} \neq \varnothing$. Then by Corollary $1 \Gamma$ must be amenable. Moreover, let $\left\{V_{n}\right\} \in W_{\Gamma}$. Since any Borel set of $\Gamma$ is $\sigma$-bounded, $V=\bigcup_{n} V_{n}$ is $\sigma$-bounded since each $V_{n}, n \in N^{+}$, is a Borel set. Consequently $\langle V\rangle$, the subgroup of $\Gamma$ generated by $V$, is also $\sigma$-bounded. But $\Gamma=\langle V\rangle$; for otherwise there is a $\gamma \in \Gamma \sim\langle V\rangle$, which clearly implies $\gamma V_{n} \cap V_{n}=\varnothing\left(n \in N^{+}\right)$, and therefore $\left|\gamma V_{n} \triangle V_{n}\right|=2\left|V_{n}\right|$ and $\left\{V_{n}\right\} \notin W_{\Gamma}$, a contradiction.

Conversely, assume $\Gamma$ is $\sigma$-compact and amenable. Let $\left\{C_{n}\right\}$ be any sequence of 
nested compact subsets of $\Gamma$ such that the union of the interiors of $C_{n}$ cover $\Gamma$. By condition (FC), for each $n \in N^{+}$we may find a Borel set $V_{n}$ of $\Gamma$ with finite nonzero measure such that $\left|\gamma V_{n} \triangle V_{n}\right|<1 / n\left|V_{n}\right|$ for all $\gamma \in K_{n}$. This clearly implies $\left\{V_{n}\right\} \in W_{\Gamma} \neq \varnothing$.

I.4. Abelian groups. We first show that $S_{\Gamma}^{0} \neq \varnothing$ for $\sigma$-compact locally compact Abelian groups, and in fact "construct" a rather special sequence $\left\{V_{n}\right\} \in S_{\Gamma}^{0}$.

THEOREM 5. If $\Gamma$ is any $\sigma$-compact locally compact Abelian group, then $S_{\Gamma}^{0} \neq \varnothing$. Moreover there exists $\left\{V_{n}\right\} \in S_{\Gamma}^{0}$ satisfying

(i) $V_{n} \subseteq V_{n+1}$ for all $n \in N^{+}$,

(ii) $\bigcup_{n \in N^{+}}$interior $\left(V_{n}\right)=\Gamma$,

(iii) Each $V_{n}$ partitions $\Gamma$, i.e. $\Gamma$ is the disjoint union of translates of $V_{n}$.

Proof. It suffices to construct the sequence $\left\{V_{n}\right\}$ of the theorem. It is clear that any $\sigma$-compact group $\Gamma$ contains an ascending sequence $\left\{K_{n}\right\}$ of compact neighborhoods of the identity such that the union of the interiors of the $K_{n}$ is $\Gamma$. Consider any such $\left\{K_{n}\right\}$ for the Abelian group we are considering, and let $H_{n}$ be the subgroup of $\Gamma$ generated by $K_{n}$. Then $H_{n}$ is a compactly generated open subgroup of $\Gamma$, and by the structure theorem for compactly generated Abelian groups there is a topological isomorphism

$$
H_{n}=Z^{a_{n}} \times R^{b_{n}} \times C_{n}
$$

where $a_{n}$ and $b_{n} \in N$ and $C_{n}$ is compact. We identify elements of the two groups under this isomorphism. Since $H_{n}$ is open in $\Gamma$ we may take Haar measure on $H_{n}$ to be the restriction of Haar measure on $\Gamma$. For all $t \in N^{+}$, we define

$$
V_{n}^{t}=\{0, \pm 1, \ldots, \pm t\}^{a_{n}} \times\{(-t, t]\}^{b_{n}} \times C_{n} .
$$

Note that $V_{n}^{t}$ partitions $\Gamma$ since the subgroup

$$
H_{n}^{t}=\{(2 t+1) Z\}^{a_{n}} \times\{2 t Z\}^{b_{n}} \times\{0\} \subseteq H_{n}
$$

has $V_{n}^{t}$ as a set of coset representatives. Also for $t_{1}, t_{2} \in N^{+}$

$$
\begin{aligned}
V_{n^{1}}^{t_{1}}+V_{n^{2}}^{t_{2}} & =V_{n^{1}+t_{2}}^{t_{1}}, \\
\left|V_{n^{2}}^{t_{1}}\right| & =\left(\frac{2 t_{2}+1}{2 t_{1}+1}\right)^{a_{n}}\left(\frac{t_{2}}{t_{1}}\right)^{b_{n}}\left|V_{n^{1}}^{t_{1}}\right| .
\end{aligned}
$$

Now since $K_{n} \subseteq H_{n}$ is compact there exists $r=r(n) \in N^{+}$such that $K_{n} \subseteq V_{n}^{r}$. But by (1.11) there also exists an $s_{0}=s_{0}(n) \in N^{+}$such that for $s \geqq s_{0},\left|V_{n}^{r}+V_{n}^{s}\right|$ $<(1+1 / n)\left|V_{n}^{s}\right|$. We now construct $V_{n}$ inductively; let $V_{1}=V_{1}^{s_{0}(1)}$, and assume $V_{k}$ has been chosen inductively for $k<n$. We choose $V_{n}=V_{n}^{t(n)}$ where $t(n)$ is subject to

(1) $t(n) \geqq s_{0}(n)$,

(2) $2 V_{n-1}=V_{n-1}+V_{n-1} \subseteq V_{n}^{t(n)}$.

(2) is true for all sufficiently large $t$ since $2 V_{n-1} \subseteq H_{n-1} \subseteq H_{n}$ and $2 V_{n-1}$ has compact closure. 
We now verify that $\left\{V_{n}\right\}$ satisfies the conditions of the theorem. (i) and (iii) are clear, and (ii) follows without difficulty from condition (2) of the inductive construction. To verify $\left\{V_{n}\right\} \in S_{\Gamma}^{0}$ let $C \neq \varnothing$ be a compact subset of $\Gamma$. Since (interior $\left(K_{n}\right): n \in N^{+}$\} was constructed to be an open cover for $\Gamma$ and since the $K_{n}$ are nested we have $C \subseteq K_{n}$ for $n \geqq n_{0}$. Hence, since $K_{n} \subseteq V_{n}^{r(n)}$

$$
1 \leqq \operatorname{Limsup}_{n \rightarrow \infty} \frac{\left|C+V_{n}\right|}{\left|V_{n}\right|} \leqq \operatorname{Limsup}_{n \rightarrow \infty} \frac{\left|V_{n}^{r(n)}+V_{n}^{t(n)}\right|}{\left|V_{n}\right|} \leqq \operatorname{Limsup}_{n \rightarrow \infty}\left(1+\frac{1}{n}\right)=1,
$$

by (1). Noting that all $V_{n}$ have compact closure, we conclude by Theorem 2 that $\left\{V_{n}\right\} \in S_{\Gamma}^{0}$.

We now prove that $\left\{V_{n}\right\} \in W_{\Gamma}$ if the sets $V_{n}$ are "well-approximated" by disjoint translates of each set which partitions $\Gamma$. More precisely:

TheOREM 6. Let $\Gamma$ be a locally compact Abelian group and $\left\{V_{n}\right\}$ a sequence of Borel sets of $\Gamma$ with finite nonzero measure. Then $\left\{V_{n}\right\} \in W_{\Gamma}$ if for every Borel set $K$ of $\Gamma$ with compact closure which partitions $\Gamma$ there exist subsets $T_{n} \subseteq \Gamma$ such that

$$
\operatorname{Lim}_{n \rightarrow \infty} \frac{\left|V_{n} \triangle \bigcup_{\tau \in T_{n}}(K+\tau)\right|}{\left|V_{n}\right|}=0
$$

and all $K+\tau, \tau \in T_{n}$ are mutually disjoint.

Proof. Fix any $\gamma \in \Gamma$, let $U$ be any compact neighborhood of $\gamma$, and let $H$ be the compactly generated open subgroup of $\Gamma$ generated by $U$. By the structure theorem there exist $a, b \in N$ and a compact group $C$ such that $H \cong Z^{a} \times R^{b} \times C$. We identify corresponding elements of these two groups. Now fix any $\varepsilon>0$. For sufficiently large $r$ the set

$$
K=K(r)=\{0, \pm 1, \ldots, \pm r\}^{a} \times((-r, r])^{b} \times C
$$

clearly satisfies $|(K+\gamma) \triangle K|<\varepsilon|K|$, where Haar measure on $H$ is taken to be the restriction of Haar measure on $\Gamma$. Moreover $K$ is a Borel set of $\Gamma$ with (finite) nonzero measure and compact closure which partitions $\Gamma$. Hence, by hypothesis there exist subsets $T_{n} \subseteq \Gamma$ such that

$$
\operatorname{Lim}_{n \rightarrow \infty} \frac{\left|V_{n} \triangle \bigcup_{\tau \in T_{n}}(K+\tau)\right|}{\left|V_{n}\right|}=0,
$$

where the union is disjoint. Using the translation invariance of Haar measure we also infer

$$
\operatorname{Lim}_{n \rightarrow \infty} \frac{\left|\left(V_{n}+\gamma\right) \triangle \bigcup_{\tau \in T_{n}}(K+\tau+\gamma)\right|}{\left|V_{n}\right|}=0 .
$$


Thus, by the metric property of $\rho(A, B)=|A \triangle B|$,

$$
\begin{aligned}
& \frac{\left|V_{n} \Delta\left(V_{n}+\gamma\right)\right|}{\left|V_{n}\right|} \leqq \frac{\left|V_{n} \Delta \bigcup_{\tau \in T_{n}}(K+\tau)\right|}{\left|V_{n}\right|}+\frac{\left|\left(\bigcup_{\tau \in T_{n}}(K+\tau)\right) \Delta\left(\bigcup_{i \in T_{n}}(K+\tau+\gamma)\right)\right|}{\left|V_{n}\right|} \\
& +\frac{\left|\bigcup_{r \in T_{n}}(K+\tau+\gamma) \Delta\left(V_{n}+\gamma\right)\right|}{\left|V_{n}\right|}
\end{aligned}
$$

and consequently

$$
\operatorname{Limsup}_{n \rightarrow \infty} \frac{\left|V_{n} \triangle\left(V_{n}+\gamma\right)\right|}{\left|V_{n}\right|} \leqq \operatorname{Limsup}_{n \rightarrow \infty} \frac{\left|\bigcup_{\tau \in T_{n}}(K+\tau) \triangle \bigcup_{\tau \in T_{n}}(K+\tau+\gamma)\right|}{\left|V_{n}\right|} .
$$

Now in general

$$
\left|\bigcup_{i \in I} A_{i} \triangle \bigcup_{i \in I} B_{i}\right| \leqq \sum_{i \in I}\left|A_{i} \triangle B_{i}\right|
$$

and therefore

$$
\begin{aligned}
\frac{\left|\bigcup_{\tau \in T_{n}}(K+\tau) \Delta \bigcup_{\tau \in T_{n}}(K+\tau+\gamma)\right|}{\left|V_{n}\right|} \leqq \frac{\sum_{\tau \in T_{n}}|(K+\tau) \Delta(K+\tau+\gamma)|}{\left|V_{n}\right|} \\
=\sum_{\tau \in T_{n}} \frac{|K \Delta(K+\gamma)|}{\left|V_{n}\right|} \leqq \frac{\varepsilon}{\left|V_{n}\right|} \sum_{\tau \in T_{n}}|K|=\varepsilon \frac{\left|\bigcup_{\tau \in T_{n}}(K+\tau)\right|}{\left|V_{n}\right|}
\end{aligned}
$$

by the construction of $K$, translation invariance, and the disjointness of $K+\tau$, $\tau \in T_{n}$. But as $n \rightarrow+\infty$

since

$$
\frac{\left|\bigcup_{r \in T_{n}}(K+\tau)\right|}{\left|V_{n}\right|} \rightarrow 1
$$

$$
\frac{\left|V_{n} \triangle \bigcup_{\tau \in T_{n}}(K+\tau)\right|}{\left|V_{n}\right|} \rightarrow 0,
$$

and we finally obtain $\operatorname{Lim} \sup _{n \rightarrow \infty}\left|V_{n} \triangle\left(V_{n}+\gamma\right)\right| /\left|V_{n}\right| \leqq e$. Since $e$ may be arbitrarily small the proof is complete.

II. Properties of $W_{\Gamma}, W_{\Gamma}^{0}, S_{\Gamma}$, and $S_{\Gamma}^{0}$. By Theorem 4 the theory is vacuous unless $\Gamma$ is $\sigma$-compact and amenable. Hence unless otherwise stated we always assume $\Gamma$ is a group of this category.

II.1. Inclusions among the basic sets. We first prove a useful proposition:

Proposition 1. If $\Gamma$ is any locally compact group, (i) $S_{\Gamma}=\varnothing \Leftrightarrow S_{\Gamma}^{0}=\varnothing$, (ii) $W_{\Gamma}=\varnothing \Leftrightarrow W_{\Gamma}^{0}=\varnothing$.

Proof. The forward implications are trivial. Conversely, assume $\left\{V_{n}\right\} \in S_{\Gamma}$ or $\left\{V_{n}\right\} \in W_{\Gamma}$. Since Haar measure is regular ([1, Theorem B, p. 254]), for each $n \in N^{+}$ we may find a compact set $V_{n}^{\prime} \subseteq V_{n}$ such that $\left|V_{n} \sim V_{n}^{\prime}\right|<1 / n\left|V_{n}\right|$. It is now easily verified that $\left\{V_{n}^{\prime}\right\} \in S_{\Gamma}^{0}$ or $\left\{V_{n}^{\prime}\right\} \in W_{\Gamma}^{0}$, respectively. 
In the following results we study the questions of relations among $W_{\Gamma}, W_{\Gamma}^{0}, S_{\Gamma}$, and $S_{\Gamma}^{0}$.

THEOREM 7. In any locally compact group $\Gamma$, (i) $W_{\Gamma}^{0} \subseteq W_{\Gamma}$, (ii) $S_{\Gamma}^{0} \subseteq S_{\Gamma}$, (iii) $S_{\Gamma}^{0} \subseteq W_{\Gamma}^{0}$, (iv) $S_{\Gamma} \subseteq W_{\Gamma}$.

Proof. (i) and (ii) are immediate from the definitions. (iv) follows since single points are compact sets, and (iii) follows from (iv) since $S_{\Gamma}^{0} \subseteq S_{\Gamma} \subseteq W_{\Gamma}$.

TheOREM 8. Suppose $S_{\Gamma}^{0} \neq \varnothing$. Then $S_{\Gamma}=S_{\Gamma}^{0} \Leftrightarrow \Gamma$ is a unimodular group.

Proof. $S_{\Gamma}^{0} \subseteq S_{\Gamma}$ always. Hence it suffices to prove $S_{\Gamma} \subseteq S_{\Gamma}^{0} \Leftrightarrow \Gamma$ is unimodular, assuming $S_{\Gamma}^{0} \neq \varnothing$.

First assume $\Gamma$ is not unimodular and let $\left\{V_{n}\right\} \in S_{\Gamma}^{0}$. Let $\left\{\gamma_{m}\right\}$ be a sequence of elements in $\Gamma$ such that,

(i) $\sum_{m{ }_{n}} \Delta\left(\gamma_{m}\right)<1 / n\left|V_{n}\right|$ for all $n \in N^{+}$,

(ii) $\Delta\left(\gamma_{n}\right)<1 / n$ for all $n \in N^{+}$,

where $\Delta$ is the modular function. Such a sequence exists because $\left|V_{n}\right|>0$ and $\Delta$ assumes arbitrarily small values on $\Gamma$. Let $E_{n}=\left\{\gamma_{m}: m \geqq n\right\}$, and set $V_{n}^{\prime}=V_{n} \cup E_{n}$. We shall show that $\left\{V_{n}^{\prime}\right\} \in S_{\Gamma} \sim S_{\Gamma}^{0}$. The set $\bar{V}_{n}^{\prime}$ is not compact since the continuous nonvanishing function $\Delta$ takes arbitrarily small values on $E_{n} \subseteq \bar{V}_{n}^{\prime}$. Therefore $\left\{V_{n}^{\prime}\right\} \notin S_{\Gamma}^{0}$. But $\left\{V_{n}^{\prime}\right\} \in S_{\Gamma}$. For, let $C \neq \varnothing$ be a compact subset of $\Gamma$. Since $\Gamma$ is not unimodular, it is certainly not discrete. Thus $\left|E_{n}\right|=0$ and hence $\left|V_{n}^{\prime}\right|=\left|V_{n}\right|$. Also

implying

$$
C V_{n}^{\prime}=C V_{n} \cup C E_{n}=C V_{n} \cup \bigcup_{m \geqq n} C \gamma_{m},
$$

Moreover,

$$
\frac{\left|C V_{n}^{\prime} \triangle C V_{n}\right|}{\left|V_{n}\right|} \leqq \sum_{m \geqq n} \frac{\left|C \gamma_{m}\right|}{\left|V_{n}\right|}=\frac{|C|}{\left|V_{n}\right|} \sum_{m \geqq n} \Delta\left(\gamma_{m}\right)<\frac{|C|}{n} \text {. }
$$

$$
\frac{\left|C V_{n}^{\prime} \triangle V_{n}^{\prime}\right|}{\left|V_{n}^{\prime}\right|}=\frac{\left|C V_{n}^{\prime} \triangle V_{n}\right|}{\left|V_{n}\right|} \leqq \frac{\left|C V_{n}^{\prime} \triangle C V_{n}\right|}{\left|V_{n}\right|}+\frac{\left|C V_{n} \triangle V_{n}\right|}{\left|V_{n}\right|}
$$

But

$$
\operatorname{Lim}_{n \rightarrow \infty} \frac{\left|C V_{n}^{\prime} \triangle C V_{n}\right|}{\left|V_{n}\right|}=\operatorname{Lim}_{n \rightarrow \infty} \frac{\left|C V_{n} \triangle V_{n}\right|}{\left|V_{n}\right|}=0
$$

and therefore $\left\{V_{n}^{\prime}\right\} \in S_{\Gamma}$.

To prove the converse, assume $\Gamma$ is unimodular and $\left\{V_{n}\right\} \in S_{\Gamma} \sim S_{\Gamma}^{0}$. For notational simplicity assume all $\bar{V}_{n}$ noncompact (if $\left\{V_{n}\right\} \in S_{\Gamma}$, any subsequence $\left\{V_{n_{i}}\right\}$ $\in S_{\Gamma}$ ). Let $C$ be any compact subset of $\Gamma$ of positive measure. We shall show $\left|C V_{n}\right|=\infty$, contradicting $\left\{V_{n}\right\} \in S_{\Gamma}$. It suffices to construct a sequence $\left\{\nu_{i}\right\} \subseteq V_{n}$ such that

$$
C \nu_{t+1} \cap \bigcup_{1 \leqq i \leqq t} C \nu_{i}=\varnothing \quad \text { for all } t \in N^{+}
$$

for this would imply

$$
\left|C V_{n}\right| \geqq\left|\bigcup_{1 \leqq i \leqq t} C \nu_{i}\right|=\sum_{1 \leqq i \leqq t}\left|C \nu_{i}\right|=t|C|
$$


since $\Gamma$ is unimodular. Therefore $\left|C V_{n}\right|=+\infty$ since $|C|>0$ and we may choose $t$ arbitrarily large. We now inductively construct $\left\{\nu_{i}\right\}$. Choose $\nu_{1} \in V_{n}$ arbitrarily, and assume $\nu_{i}$ for $1 \leqq i \leqq t$ have already been chosen. Let $C_{t}=\bigcup_{1 \leqq i \leqq t} C \nu_{i}$. Then $C^{-1} C_{t}$ is compact. Since $\bar{V}_{n}$ is not compact $V_{n} \nsubseteq C^{-1} C_{t}$. Then (*) holds if $\nu_{t+1}$ is chosen to be any point in $V_{n} \sim C^{-1} C_{t} \neq \varnothing$, and the proof is completed.

The following lemma, which is of independent interest, is useful in the proof of the next theorem.

LEMMA 3. Let $\Gamma$ be a noncompact locally compact group with modular function $\Delta$ and let $R$ be a relatively compact subset of $\Gamma$. Then there exists an infinite subset $\mathscr{D}=\left\{\gamma_{i}\right\}$ in $\Gamma$ such that the $R \gamma_{i}$ are pairwise disjoint and $\Delta\left(\gamma_{i}\right) \geqq 1$ for all $i$.

Proof. Obviously we may assume that $R$ is compact. Let $\Gamma_{+}=\{\gamma \in \Gamma \mid \Delta(\gamma) \geqq 1\}$ and let $\mathscr{D}$ be maximal in $\Gamma_{+}$such that the sets $R \gamma, \gamma \in \mathscr{D}$, are pairwise disjoint. This implies $R \mu \cap R \mathscr{D} \neq \varnothing$ for every $\mu \in \Gamma_{+}$. Hence $\Gamma_{+} \subset R^{-1} R \mathscr{D}$ and so $\mathscr{D}$ cannot be finite (or relatively compact) because $\Gamma_{+}$is not compact (observe $\Gamma=\Gamma_{+} \cup \Gamma_{+}^{-1}$ ).

REMARK. If $\left\{\gamma_{i}\right\} \subset \Gamma$ is such that $\left\{\Delta\left(\gamma_{i}\right)\right\}$ is a discrete subset of the positive real numbers, in particular if $\lim \Delta\left(\gamma_{i}\right)=\infty$, then $\left\{\gamma_{i}\right\}$ is closed and discrete because $\Delta$ is continuous. If $R$ in Lemma 3 is chosen with nonvoid interior, the set $\mathscr{D}$ necessarily is closed and discrete in $\Gamma_{+}$.

THEOREM 9. Let $W_{\Gamma} \neq \varnothing$. The following properties are equivalent:

(i) $\Gamma$ is compact or discrete.

(ii) $W_{\Gamma}=W_{\Gamma}^{0}$.

(iii) $S_{\Gamma}=W_{\Gamma}$.

(iv) $S_{\Gamma}^{0}=W_{\Gamma}^{0}$.

Moreover, if $\Gamma$ is neither compact nor discrete, there exists $\left\{V_{n}^{\prime}\right\} \in W_{\Gamma}$ such that $\left|C V_{n}^{\prime}\right|=+\infty$ for all $n \in N^{+}$and any Borel set $C$ of positive measure.

Proof. If $\Gamma$ is discrete $W_{\Gamma}=W_{\Gamma}^{0}$ and $S_{\Gamma}=S_{\Gamma}^{0}$ immediately from the definition. Also by Theorem 2 and Theorem 1 (ii) we conclude $S_{\Gamma}=W_{\Gamma}$. If $\Gamma$ is compact, trivially $S_{\Gamma}=S_{\Gamma}^{0}$ and $W_{\Gamma}=W_{\Gamma}^{0}$. To show that $S_{\Gamma}=W_{\Gamma}$ we first assume Haar measure on $\Gamma$ is normalized so that $|\Gamma|=1$. Let $\left\{V_{n}\right\} \in W_{\Gamma}$. We shall show that this implies $\operatorname{Lim}_{n \rightarrow \infty}\left|V_{n}\right|=1$, from which we clearly conclude $\left\{V_{n}\right\} \in S_{\Gamma}$. For $\left\{V_{n}\right\} \in W_{\Gamma}$ implies

$$
\operatorname{Lim}_{n \rightarrow \infty} \frac{\left|\gamma V_{n} \cap V_{n}\right|}{\left|V_{n}\right|}=\operatorname{Lim}_{n \rightarrow \infty} \frac{\left|V_{n}\right|-\frac{1}{2}\left|\gamma V_{n} \triangle V_{n}\right|}{\left|V_{n}\right|}=1 \quad \text { for all } \gamma \in \Gamma \text {. }
$$

Hence upon recalling the formula $\int_{\Gamma}|\gamma A \cap B| d \gamma=|A| \cdot\left|B^{-1}\right|$, by dominated convergence we obtain

$$
1=\operatorname{Lim}_{n \rightarrow \infty} \int_{\Gamma} \frac{\left|\gamma V_{n} \cap V_{n}\right| d \gamma}{\left|V_{n}\right|}=\operatorname{Lim}_{n \rightarrow \infty} \frac{\left|V_{n}\right| \cdot\left|V_{n}^{-1}\right|}{\left|V_{n}\right|}=\operatorname{Lim}_{n \rightarrow \infty}\left|V_{n}\right| .
$$

Conversely, if $\Gamma$ is noncompact and nondiscrete let $R$ be any compact neighborhood of the unit in $\Gamma$ and let $\mathscr{D}=\left\{\gamma_{1}, \gamma_{2}, \ldots\right\} \subset \Gamma_{+}$be a sequence as guaranteed by Lemma 3. Let $\left\{V_{n}\right\} \in W_{\Gamma}$ and define $V_{n}^{\prime}=V_{n} \cup \mathscr{D}$. 
(i) Clearly $\gamma V_{n}^{\prime} \triangle V_{n}^{\prime} \subseteq\left(\gamma V_{n} \triangle V_{n}\right) \cup(\mathscr{D} \cup \gamma \mathscr{D})$, and consequently

$$
\frac{\left|\gamma V_{n}^{\prime} \triangle \dot{V}_{n}^{\prime}\right|}{\left|V_{n}^{\prime}\right|} \leqq \frac{\left|\gamma V_{n} \triangle V_{n}\right|}{\left|V_{n}\right|}
$$

since countable sets have zero measure in nondiscrete groups, and therefore $\left\{V_{n}^{\prime}\right\} \in W_{\Gamma}$. Since $\mathscr{D} \subset \bar{V}_{n}^{\prime}$, we have $\left\{V_{n}^{\prime}\right\} \notin W_{\Gamma}^{0}$ and thus $W_{\Gamma} \neq W_{\Gamma}^{0}$.

(ii) Continuing from (i) we shall also show that $\left\{V_{n}^{\prime}\right\} \notin S_{\Gamma}$, and in fact $\left|C V_{n}^{\prime}\right|=\infty$ for any Borel set $C$ in $\Gamma$ of positive measure. First, there exists $\nu \in \Gamma$ with $|\nu C \cap R|$ >0. It follows that

$$
\begin{aligned}
\left|C V_{n}^{\prime}\right| & =\left|\nu C V_{n}^{\prime}\right| \geqq|(\nu C \cap R) \mathscr{D}|=\sum_{1}^{\infty}\left|(\nu C \cap R) \gamma_{i}\right| \\
& =\sum_{1}^{\infty} \Delta\left(\gamma_{i}\right)|\nu C \cap R|=\infty
\end{aligned}
$$

since $\Delta\left(\gamma_{i}\right) \geqq 1$.

(iii) If $W_{\Gamma}^{0}=\varnothing$, then $W_{\Gamma}=\varnothing$ by Proposition 1. Hence assume $\left\{V_{n}\right\} \in W_{\Gamma}^{0}$. If $\left\{t_{n}\right\}$ is any sequence of natural numbers and we set $V_{n}^{\prime}=V_{n} \cup\left\{\gamma_{i} \in \mathscr{D} \mid 1 \leqq i \leqq t_{n}\right\}$ we clearly have $\left\{V_{n}^{\prime}\right\} \in W_{\Gamma}^{0}$. But as in the above paragraph, if $C$ is any Borel set of $\Gamma$ of positive measure $|C \mathscr{D}|=\infty$ and therefore if we choose $t_{n}$ increasing sufficiently rapidly (depending on our choice of $V_{n}$ and $C$ ) we may guarantee $\left|C V_{n}^{\prime}\right| /\left|V_{n}^{\prime}\right| \rightarrow \infty$ as $n \rightarrow \infty$. We conclude $\left\{V_{n}^{\prime}\right\} \notin S_{\Gamma}^{0}$ by Theorem 2 .

II.2. Closure properties of the basic sets. We generically let $R_{\Gamma}$ denote any of the four sets $W_{\Gamma}, W_{\Gamma}^{0}, S_{\Gamma}, S_{\Gamma}^{0}$. Trivially, if $\left\{V_{n}\right\} \in R_{\Gamma}$ any subsequence $\left\{V_{n}\right\}$ is also in $R_{\Gamma}$ as well as any sequence $\left\{U_{n}\right\}$ of Borel sets of $\Gamma$ with finite nonzero measure with only finitely many $U_{n} \neq V_{n}$. Also, if $\left\{V_{n}^{(i)}\right\} \in R_{\Gamma}$ for $1 \leqq i \leqq t$ and $\left\{V_{m}^{*}\right\}$ is an arbitrary sequence such that for all $m \in N^{+}, V_{m}^{*}=V_{n}^{(i)}$ for some $n \in N^{+}, 1 \leqq i \leqq t$, and every $V_{n}^{(i)}$ occurs only finitely often as a $V_{m}^{*}$, then $\left\{V_{m}^{*}\right\} \in R_{\Gamma}$. The following theorem lists further closure properties of $R_{\Gamma}$.

THEOREM 10. (i) If $\left\{V_{n}\right\} \dot{\in} R_{\Gamma}$ and $\left\{\gamma_{n}\right\}$ is any sequence of elements of $\Gamma$, then $\left\{V_{n} \gamma_{n}\right\} \in R_{\Gamma}$.

(ii) If $\left\{U_{n}\right\}$ and $\left\{V_{n}\right\} \in R_{\Gamma}$, then $\left\{U_{n} \cup V_{n}\right\} \in R_{\Gamma}$.

(iii) If $\left\{V_{n}\right\}$ is a sequence of Borel sets of $\Gamma$ with finite nonzero measure such that for every $\varepsilon>0$ there exists $a\left\{V_{n}^{\varepsilon}\right\} \in W_{\Gamma}$ satisfying $\operatorname{Lim} \sup _{n \rightarrow \infty}\left|V_{n} \triangle V_{n}^{\varepsilon}\right| /\left|V_{n}\right|<\varepsilon$, then $\left\{V_{n}\right\} \in W_{\Gamma}$.

(iv) If $\left\{V_{n}\right\}$ is a sequence of Borel sets of $\Gamma$ with finite nonzero measure such that for every $\varepsilon>0$ there exists $a\left\{V_{n}^{\varepsilon}\right\} \in S_{\Gamma}$ satisfying $V_{n} \subseteq V_{n}^{\varepsilon}$ and $\operatorname{Lim} \sup _{n \rightarrow \infty}\left|V_{n}^{\varepsilon} \sim V_{n}\right| /\left|V_{n}\right|$ $<\varepsilon$, then $\left\{V_{n}\right\} \in S_{\Gamma}$.

Proof. (i) A Borel set $V$ of $\Gamma$ has finite nonzero measure or compact closure if and only if $V \gamma$ does, where $\gamma$ is any element of $\Gamma$. Also, if $C \neq \varnothing$ is a compact set, 
$|C V \triangle V| /|V|=|C(V \gamma) \triangle(V \gamma)| /|V \gamma|$ and (i) clearly follows. (ii) follows since the union of two Borel sets with compact closure has compact closure and

$$
\left|C\left(U_{n} \cup V_{n}\right) \triangle\left(U_{n} \cup V_{n}\right)\right| \leqq\left|C U_{n} \triangle U_{n}\right|+\left|C V_{n} \triangle V_{n}\right|
$$

To prove (iii), we apply the metric property of $\rho(A, B)=|A \triangle B|$ and infer

$$
\left|\gamma V_{n} \triangle V_{n}\right| \leqq\left|\gamma V_{n} \triangle \gamma V_{n}^{\varepsilon}\right|+\left|\gamma V_{n}^{\varepsilon} \triangle V_{n}^{\varepsilon}\right|+\left|V_{n}^{\varepsilon} \triangle V_{n}\right|
$$

\section{Consequently}

$$
\underset{n \rightarrow \infty}{\operatorname{Limsup}} \frac{\left|\gamma V_{n} \triangle V_{n}\right|}{\left|V_{n}\right|} \leqq \operatorname{Limsup}_{n \rightarrow \infty} \frac{\left(\left|\gamma V_{n} \triangle \gamma V_{n}^{\varepsilon}\right|+\left|\gamma V_{n}^{\varepsilon} \triangle V_{n}^{\varepsilon}\right|+\left|V_{n}^{\varepsilon} \triangle V_{n}\right|\right)}{\left|V_{n}\right|}<2 \varepsilon,
$$

and since $\varepsilon>0$ is arbitrary we have $\left\{V_{n}\right\} \in W_{\Gamma}$.

Finally, to prove (iv) we note that $V_{n} \subseteq V_{n}^{\varepsilon}$ implies

$$
\begin{aligned}
C V_{n} \triangle V_{n} & \subseteq\left(C V_{n} \triangle C V_{n}^{\varepsilon}\right) \cup\left(C V_{n}^{\varepsilon} \triangle V_{n}^{\varepsilon}\right) \cup\left(V_{n}^{\varepsilon} \triangle V_{n}\right) \\
& =\left(C V_{n}^{\varepsilon} \sim C V_{n}\right) \cup\left(C V_{n}^{\varepsilon} \triangle V_{n}^{\varepsilon}\right) \cup\left(V_{n}^{\varepsilon} \sim V_{n}\right) .
\end{aligned}
$$

But

$$
\begin{aligned}
\left|C V_{n}^{\varepsilon} \sim C V_{n}\right| & =\left|C V_{n}^{\varepsilon}\right|-\left|C V_{n}\right| \leqq\left|C V_{n}^{\varepsilon}\right|-\left|V_{n}\right| \\
& \leqq\left|C V_{n}^{\varepsilon} \triangle V_{n}^{\varepsilon}\right|+\left|V_{n}^{\varepsilon}\right|-\left|V_{n}\right|=\left|C V_{n}^{\varepsilon} \triangle V_{n}^{\varepsilon}\right|+\left|V_{n}^{\varepsilon} \sim V_{n}\right|
\end{aligned}
$$

and therefore

$$
\operatorname{Limsup}_{n \rightarrow \infty} \frac{\left|C V_{n} \triangle V_{n}\right|}{\left|V_{n}\right|} \leqq \operatorname{Limsup}_{n \rightarrow \infty} \frac{2\left(\left|C V_{n}^{\varepsilon} \triangle V_{n}^{\varepsilon}\right|+\left|V_{n}^{\varepsilon} \sim V_{n}\right|\right)}{\left|V_{n}\right|}<2 \varepsilon .
$$

Hence $\left\{V_{n}\right\} \in S_{\Gamma}$.

By Theorem 10 (i) we see that any sequence $\left\{V_{n}\right\} \in R_{\Gamma}$ can be arbitrarily translated on the right and the resulting sequence $\left\{V_{n} \gamma_{n}\right\}$ remains in $R_{\Gamma}$. We now consider the more delicate problem of left translation, and prove the following theorem:

THEOREM 11. $\left\{V_{n}\right\} \in W_{\Gamma}$ implies $\left\{\gamma_{n} V_{n}\right\} \in W_{\Gamma}$ for any sequence of elements $\left\{\gamma_{n}\right\} \subseteq \Gamma$ if and only if every conjugacy class $\alpha^{\Gamma}=\left\{\gamma^{-1} \alpha \gamma: \gamma \in \Gamma\right\}$ in $\Gamma$ has compact closure $\mathrm{cl}\left(\alpha^{\Gamma}\right)$.

Proof. Assume $\operatorname{cl}\left(\alpha^{\Gamma}\right)$ is compact, and let $\left\{\gamma_{n}\right\} \subseteq \Gamma$. Then

$$
\operatorname{Lim}_{n \rightarrow \infty} \frac{\left|\alpha\left(\gamma_{n} V_{n}\right) \triangle\left(\gamma_{n} V_{n}\right)\right|}{\left|\gamma_{n} V_{n}\right|}=\operatorname{Lim}_{n \rightarrow \infty} \frac{\left|\left(\gamma_{n}^{-1} \alpha \gamma_{n}\right) V_{n} \triangle V_{n}\right|}{\left|V_{n}\right|}=0
$$

by Corollary 2, since $\gamma_{n}^{-1} \alpha \gamma_{n}=\gamma_{n}^{\prime} \in \alpha^{\Gamma}$ for all $n \in N^{+}$. Conversely, assume cl $\left(\alpha^{\Gamma}\right)$ noncompact for some $\alpha \in \Gamma$ and let $\left\{V_{n}\right\} \in W_{\Gamma}^{0}\left(\subseteq W_{\Gamma}\right)$. Construct $\left\{\gamma_{n}\right\}$ subject to the condition $\gamma_{n}^{-1} \alpha \gamma_{n} \notin V_{n} \cdot V_{n}^{-1}$, which is feasible since we may assume all $\operatorname{cl}\left(V_{n}\right)$ compact, hence all $\mathrm{cl}\left(V_{n} \cdot V_{n}^{-1}\right)$ compact, and $\mathrm{cl}\left(\alpha^{\Gamma}\right)$ is noncompact. Then for this sequence $\left\{\gamma_{n}\right\}$ we have

$$
\alpha \gamma_{n} V_{n} \triangle \gamma_{n} V_{n}=\gamma_{n}\left\{\left(\gamma_{n}^{-1} \alpha \gamma_{n}\right) V_{n} \triangle V_{n}\right\}=\alpha \gamma_{n} V_{n} \cup \gamma_{n} V_{n}
$$


and hence $\left|\alpha \gamma_{n} V_{n} \triangle \gamma_{n} V_{n}\right| /\left|V_{n}\right|=2$ and $\left\{\gamma_{n} V_{n}\right\} \notin W_{\Gamma}$. We now prove a result of independent interest which will also be used in the proof of Theorem 13.

THEOREM 12. Let $\left\{V_{n}\right\}$ be a sequence of Borel sets of finite nonzero measure in any locally compact group $\Gamma$ satisfying

$$
\operatorname{Lim}_{n \rightarrow \infty}\left|\gamma V_{n} \triangle V_{n}\right| /\left|V_{n}\right|=0 \text { for all } \gamma \in U,
$$

where $U$ is a measurable subset of $\Gamma$. Then $\operatorname{Lim}_{\inf _{n \rightarrow \infty}}\left|V_{n}^{-1}\right| \geqq|U|$.

Proof. First assume $|U|<+\infty$. Then by (*) $\operatorname{Lim}_{n \rightarrow \infty}\left|\gamma V_{n} \cap V_{n}\right| /\left|V_{n}\right|=1$ for all $\gamma \in U$, and hence by dominated convergence

$$
\operatorname{Lim}_{n \rightarrow \infty} \int_{U} \frac{\left|\gamma V_{n} \cap V_{n}\right|}{\left|V_{n}\right|} d \gamma=|U|
$$

But by Fubini's Theorem

$$
\begin{aligned}
\int_{U} \frac{\left|\gamma V_{n} \cap V_{n}\right|}{\left|V_{n}\right|} d \gamma & =\frac{1}{\left|V_{n}\right|} \int_{U}\left[\int_{V_{n}} I_{V_{n}}\left(\gamma^{-1} \tau\right) d \tau\right] d \gamma \\
& =\frac{1}{\left|V_{n}\right|} \int_{V_{n}}\left[\int_{U} I_{V_{n}}\left(\gamma^{-1} \tau\right) d \gamma\right] d \tau=\frac{1}{\left|V_{n}\right|} \int_{V_{n}}\left|U \cap \tau V_{n}^{-1}\right| d \tau \\
& \leqq \frac{1}{\left|V_{n}\right|} \int_{V_{n}}\left|\tau V_{n}^{-1}\right| d \tau=\left|V_{n}^{-1}\right|
\end{aligned}
$$

and therefore $\operatorname{Lim}_{\inf } \ln _{n \rightarrow \infty}\left|V_{n}^{-1}\right| \geqq|U|$.

If $|U|=+\infty$ we may find measurable subsets of $U$ with arbitrarily large finite measure, and since (*) trivially is satisfied on any subset of $U$, we conclude $\operatorname{Lim}_{n \rightarrow \infty}\left|V_{n}^{-1}\right|=+\infty$ by the estimate above.

Corollary 4. If $\Gamma$ is not compact, then

(i) $\left\{V_{n}\right\} \in W_{\Gamma}$ implies $\operatorname{Lim}_{n \rightarrow \infty}\left|V_{n}^{-1}\right|=+\infty$.

(ii) If $W_{\Gamma} \neq \varnothing, \operatorname{Lim}_{n \rightarrow \infty}\left|V_{n}\right|=+\infty$ for all $\left\{V_{n}\right\} \in W_{\Gamma}$ if and only if $\Gamma$ is unimodular. In fact, if $\Gamma$ is not unimodular and $\varepsilon>0$ there exists a sequence $\left\{V_{n}^{\varepsilon}\right\} \in W_{\Gamma}$ such that $\sum_{n}\left|V_{n}^{\varepsilon}\right|<\varepsilon$.

Proof. (i) is immediate since $\left\{V_{n}\right\} \in W_{\Gamma}$ implies (*) is satisfied for $U=\Gamma$ and $|\Gamma|$ $=+\infty$ since $\Gamma$ is assumed noncompact.

(ii): If $\Gamma$ is unimodular $\left|V_{n}^{-1}\right|=\left|V_{n}\right|$ and therefore $\left\{V_{n}\right\} \in W_{\Gamma}$ implies $\operatorname{Lim}_{n \rightarrow \infty}\left|V_{n}\right|$ $=+\infty$ by (i). On the other hand, if $\Gamma$ is not unimodular and $\left\{V_{n}\right\}$ is any sequence in $W_{\Gamma}$ then $\left\{V_{n} \gamma_{n}\right\} \in W_{\Gamma}$ for any sequence $\left\{\gamma_{n}\right\} \subseteq \Gamma$ by Theorem 10 (i). Now since $\Gamma$ is not unimodular, for each $n \in N^{+}$we may choose $\gamma_{n}^{\delta} \in \Gamma$ such that $\Delta\left(\gamma_{n}^{\varepsilon}\right)<\varepsilon /\left(2^{n}\left|V_{n}\right|\right)$. Thus if we set $V_{n}^{\varepsilon}=V_{n} \gamma_{n}^{\varepsilon},\left\{V_{n}^{\varepsilon}\right\} \in W_{\Gamma}$ and

$$
\sum_{n}\left|V_{n}^{\varepsilon}\right|=\sum_{n}\left|V_{n} \gamma_{n}^{\varepsilon}\right|=\sum_{n}\left|V_{n}\right| \Delta\left(\gamma_{n}^{\varepsilon}\right)<\sum \varepsilon / 2^{n}=\varepsilon
$$

as asserted. 
THEOREM 13. If $\Gamma$ is a nonunimodular $\sigma$-compact amenable group,

$$
C(\Gamma)=\left\{\alpha \in \Gamma: \operatorname{cl}\left(\alpha^{\Gamma}\right) \text { is compact }\right\}
$$

has measure zero. Moreover, if $\left\{\gamma_{n}\right\}$ is any sequence of elements of $\Gamma$ for which $\left\{\Delta\left(\gamma_{n}\right)\right\}$ is unbounded

$$
C\left(\left\{\gamma_{n}\right\}\right)=\left\{\alpha \in \Gamma: \operatorname{cl}\left(\left\{\gamma_{n}^{-1} \alpha \gamma_{n}\right\}\right) \text { is compact }\right\}
$$

has measure zero.

Proof. Since $C(\Gamma) \subseteq C\left(\left\{\gamma_{n}\right\}\right)$ it suffices to prove the latter statement. First note that $C\left(\left\{\gamma_{n}\right\}\right)$ is measurable: for if $\left\{K_{i}\right\}$ is any nested sequence of compacta in $\Gamma$ whose interiors cover $\Gamma$ (such sequences clearly exist in arbitrary $\sigma$-compact groups), we have $C\left(\left\{\gamma_{n}\right\}\right)=\bigcup_{i}\left\{\bigcap_{n} \gamma_{n} K_{i} \gamma_{n}^{-1}\right\}$ because cl $\left(\left\{\gamma_{n}^{-1} \alpha \gamma_{n}\right\}\right)$ is compact if and only if $\left\{\gamma_{n}^{-1} \alpha \gamma_{n}\right\} \subseteq K_{i}$ for some $i$ if and only if $\alpha \in \bigcap_{n} \gamma_{n} K_{i} \gamma_{n}^{-1}$ for some $i$. Now let $\left\{V_{n}\right\} \in W_{\Gamma}^{0}$ and set $V_{n}^{\prime}=\gamma_{n} V_{n}$. Let $\alpha \in C\left(\left\{\gamma_{n}\right\}\right)$, and consider $\left|\alpha V_{n}^{\prime} \triangle V_{n}^{\prime}\right| /\left|V_{n}^{\prime}\right|$ $=\left|\left(\gamma_{n}^{-1} \alpha \gamma_{n}\right) V_{n} \triangle V_{n}\right| /\left|V_{n}\right|:$ once more by Corollary 2 we see that this quotient tends to zero as $n \rightarrow+\infty$. Hence by Theorem 12,

$$
\left|C\left(\left\{\gamma_{n}\right\}\right)\right| \leqq \operatorname{Liminf}_{n \rightarrow+\infty}\left|V_{n}^{\prime-1}\right|=\operatorname{Liminf}_{n \rightarrow \infty}\left|V_{n}^{-1} \gamma_{n}^{-1}\right|=\operatorname{Liminf}_{n \rightarrow \infty}\left|V_{n}^{-1}\right| \triangle\left(\gamma_{n}^{-1}\right) .
$$

Now the left hand side of this inequality is independent of our choice of $\left\{V_{n}\right\}$, and we may clearly assume $\Delta\left(\gamma_{n}^{-1}\right) \rightarrow 0$. Hence for an appropriate choice of $\left\{V_{n}\right\}$ (e.g., "repeating" terms of any $\left\{V_{n}\right\}$ with appropriate increasing "multiplicites") the right hand side is zero, and the proof is complete.

COROllaRY 5. If $\Gamma$ is a nonunimodular amenable $\sigma$-compact group and $\left\{V_{n}\right\} \in W_{\Gamma}$ then $\left\{\gamma_{n} V_{n}\right\} \notin W_{\Gamma}$ for some sequence $\left\{\gamma_{n}\right\} \subseteq \Gamma$.

Proof. Theorem 13 and Theorem 11.

1I.3. The approximation theorem. In Theorem 9 we proved that if $W_{\Gamma} \neq \varnothing$ then $W_{\Gamma}^{0} \neq S_{\Gamma}^{0}$ unless $\Gamma$ is compact or discrete. In this section we show that nevertheless $S_{\Gamma}^{0}$ is in a sense dense in $W_{\Gamma}$, and apply this result to obtain a characterization of $W_{\Gamma}$. We first make a definition:

Definition 3. Let $\Gamma$ be any group and let $K \subseteq \Gamma$ be any subset of $\Gamma$. For any subset $T \subseteq \Gamma$ we define $C(K, T)$ to be the maximum number of $K \tau, \tau \in T$ which intersect, possibly $+\infty . I_{R}(\Gamma, K)$, the right index of $K$ in $\Gamma$, is defined by

$$
I_{R}(\Gamma, K)=\operatorname{Inf}\left\{C(K, T): T \subseteq \Gamma, \Gamma=\bigcup_{\tau \in T} K \tau\right\}
$$

We need the following result of Greenleaf and the author [2]:

THEOREM 14. If $\Gamma$ is any locally compact group, there exists a Borel set $K \subseteq \Gamma$ with compact closure and nonvoid interior such that $I_{R}(\Gamma, K)<+\infty$.

We next prove: 
Proposition 2. If $\Gamma$ is any topological group and there exists a subset $K \subseteq \Gamma$ with compact closure, nonvoid interior, and finite right index in $\Gamma$, then every subset $C \subseteq \Gamma$ with compact closure and nonvoid interior has finite right index.

Proof. Let $\Gamma=\bigcup_{\imath \in T} K \tau$ be such that at most $n$ right translates $K \tau, \tau \in T$ intersect. Since $K$ has compact closure and $C$.has nonvoid interior, we may cover $K$ by finitely many right translates of $C$, say

$$
K \subseteq C \alpha_{1} \cup C \alpha_{2} \cup \cdots \cup C \alpha_{r}=C^{\prime} .
$$

But $C^{\prime}$ has compact closure since $C$ does, and $K$ has nonvoid interior and therefore we may cover $C^{\prime}$ by finitely many left translates of $K$, say

$$
C^{\prime} \subseteq \beta_{1} K \cup \beta_{2} K \cup \cdots \cup \beta_{s} K=K^{\prime} .
$$

We shall prove that this implies $I_{R}(\Gamma, C) \leqq n r s$. For consider the decomposition

$$
\Gamma=\bigcup_{\tau \in T} K \tau=\bigcup_{\tau \in T}\left(\left(C \alpha_{1} \tau\right) \cup\left(C \alpha_{2} \tau\right) \cup \cdots \cup\left(C \alpha_{r} \tau\right)\right),
$$

and assume more than $n r s$ of the $C \alpha_{i} \tau, 1 \leqq i \leqq r, \tau \in T$, intersect. This implies more than $n s$ different $\tau$ must occur. Hence more than $n s$ of the $C^{\prime} \tau, \tau \in T$ intersect, and consequently $m>n s$ of the $K^{\prime} \tau, \tau \in T$ intersect, say $\bigcap_{1 \leqq i \leqq m} K^{\prime} \tau_{i} \neq \varnothing$. Let $\gamma_{0}$ be any element of this intersection, and for each $i, 1 \leqq i \leqq m$, let $u=u(i)$ be some integer $1 \leqq u \leqq s$ for which $\gamma_{0} \in \beta_{u} K \tau_{i}$. Hence $u(i)$ must take some value $u_{0}$ more than $n$ times, say $u\left(i_{1}\right)=u\left(i_{2}\right)=\cdots=u\left(i_{n^{\prime}}\right)=u_{0}$, where $n^{\prime}>n$. But this implies

$$
\gamma_{0} \in \bigcap_{1 \leqq j \leqq n^{\prime}} \beta_{u_{0}} K \tau_{i j}, \beta_{u_{0}}^{-1} \gamma_{0} \in \bigcap_{1 \leqq j \leqq n^{\prime}} K \tau_{i,} \neq \varnothing .
$$

Combining the two preceding results we obtain

Proposition 3. If $\Gamma$ is any locally compact group, every Borel set with compact closure and nonvoid interior has finite right index in $\Gamma$.

We now prove a lemma which is critical in the proof of The Approximation Theorem:

LEMMA 4. Let $\Gamma$ be a locally compact group and let $K$ be a Borel set of $\Gamma$ containing $e$ with compact closure and nonvoid interior. Then if $\left\{V_{n}\right\} \in W_{\Gamma}^{0}$ and $0<\varepsilon<1$, there exists a sequence $\left\{V_{n}^{\prime}\right\}$ of Borel sets of $\Gamma$ such that

(i) $V_{n}^{\prime} \subseteq V_{n},\left|V_{n} \sim V_{n}^{\prime}\right| \leqq \varepsilon\left|V_{n}\right|$ for all $n \in N^{+}$.

(ii) $\operatorname{Lim} \sup _{n \rightarrow \infty}\left|K V_{n}^{\prime}\right| /\left|V_{n}^{\prime}\right| \leqq 1 /(1-\varepsilon)$.

Proof. $|K|>0$ since $K$ has nonvoid interior. Also $K^{\prime}=K \cdot K \cdot K^{-1}$ is a set with compact closure. For any $n \in N^{+}$and $\delta>0$ we define the Borel set $E_{n}(\delta)$ by

$$
E_{n}(\delta)=\left\{\gamma \in V_{n}:\left|K^{\prime} \sim V_{n} \gamma^{-1}\right|>\delta\right\},
$$

and let

$$
\delta_{n}=\operatorname{Inf}\left\{\delta:\left|E_{n}(\delta)\right| \leqq \varepsilon\left|V_{n}\right|\right\}
$$


Therefore $E_{n}(\delta)>\varepsilon\left|V_{n}\right|$ if $\delta<\delta_{n}$, and $\left|E_{n}\left(\delta_{n}\right)\right| \leqq \varepsilon\left|V_{n}\right|$ since Haar measure is continuous.

We shall now prove that $V_{n}^{\prime}=V_{n} \sim E_{n}\left(\delta_{n}\right)$ satisfies conditions (i) and (ii) of the lemma. Clearly (i) is true. As a first step in proving (ii) we shall first show $\operatorname{Lim}_{n \rightarrow \infty} \delta_{n}=0$. For assume $\operatorname{Lim} \sup _{n \rightarrow \infty} \delta_{n}>0$; then without loss of generality we may assume there exists an $\eta>0$ and an $n_{0}$ such that for $n \geqq n_{0}$ we have $\eta<\delta_{n}$ and therefore

$$
\left|E_{n}(\eta)\right|>\varepsilon\left|V_{n}\right| \text {. }
$$

This will result in a contradiction. For $\gamma \in E_{n}(\eta), K_{y}=K_{\gamma}(n)=K^{\prime} \sim V_{n} \gamma^{-1} \subseteq K^{\prime}$ clearly satisfies

$$
\left|K_{\gamma}\right|>\eta, \quad K_{\gamma} \gamma \cap V_{n}=\varnothing .
$$

Also, for each $\alpha \in K^{\prime}$, let

We then have

$$
S_{\alpha}=S_{\alpha}(n)=\left\{\gamma \in E_{n}(\eta): \alpha \in K_{\gamma}\right\}
$$

$$
\int_{E_{n}(\eta)}\left|K_{y}\right| d \gamma=\int_{K^{\prime}}\left|S_{\alpha}\right| d \alpha
$$

since upon application of Fubini's Theorem

$$
\begin{aligned}
\int_{E_{n}(\eta)}\left|K_{y}\right| d \gamma & =\int_{E_{n}(\eta)}\left[\int_{K^{\prime}} I_{K_{y}}(\alpha) d \alpha\right] d \gamma=\int_{K^{\prime}}\left[\int_{E_{n}(\eta)} I_{K_{\gamma}}(\alpha) d \gamma\right] d \alpha \\
& =\int_{K^{\prime}}\left|\left\{\gamma \in E_{n}(\eta): \alpha \in K_{y}\right\}\right| d \alpha=\int_{K^{\prime}}\left|S_{\alpha}\right| d \alpha
\end{aligned}
$$

Now $\gamma \in S_{\alpha}$ implies $\alpha \in K_{\gamma}=K^{\prime} \sim V_{n} \gamma^{-1}$ and consequently $\alpha \gamma \notin V_{n}$ and thus $\alpha S_{\alpha} \cap V_{n}=\varnothing$. But $S_{\alpha} \subseteq V_{n}$ for $\alpha \in K^{\prime}$ and therefore $\alpha V_{n} \cap V_{n}=\alpha\left(V_{n} \sim S_{\alpha}\right) \cap V_{n}$. But $\left\{V_{n}\right\} \in W_{\Gamma}$ implies

$$
\begin{aligned}
1 & =\operatorname{Lim}_{n \rightarrow \infty} \frac{\left|\alpha V_{n} \cap V_{n}\right|}{\left|V_{n}\right|}=\operatorname{Lim}_{n \rightarrow \infty} \frac{\left|\alpha\left(V_{n} \sim S_{\alpha}\right) \cap V_{n}\right|}{\left|V_{n}\right|} \leqq \operatorname{Liminf}_{n \rightarrow \infty} \frac{\left|\alpha\left(V_{n} \sim S_{\alpha}\right)\right|}{\left|V_{n}\right|} \\
& =\operatorname{Liminf}_{n \rightarrow \infty} \frac{\left|V_{n}\right|-\left|S_{\alpha}\right|}{\left|V_{n}\right|}=1-\operatorname{Limsup}_{n \rightarrow \infty} \frac{\left|S_{\alpha}\right|}{\left|V_{n}\right|}
\end{aligned}
$$

and hence we may conclude $\operatorname{Lim}_{n \rightarrow \infty}\left|S_{\alpha}(n)\right| /\left|V_{n}\right|=0$ for $\alpha \in K^{\prime}$. Therefore by dominated convergence

$$
\operatorname{Lim}_{n \rightarrow \infty} \int_{\Sigma^{\prime}} \frac{\left|S_{\alpha}\right|}{\left|V_{n}\right|} d \alpha=0
$$

By (2.1) to (2.4), $\left|E_{n}(\eta)\right|>\varepsilon\left|V_{n}\right|,\left|K_{y}(n)\right|>\eta$ on $E_{n}(\eta)$, and

$$
0=\operatorname{Lim}_{n \rightarrow \infty} \int_{K^{\prime}} \frac{\left|S_{\alpha}\right|}{\left|V_{n}\right|} d \alpha=\operatorname{Lim}_{n \rightarrow \infty} \frac{1}{\left|V_{n}\right|} \int_{E_{n}(n)}\left|K_{\gamma}(n)\right| d \gamma \geqq \operatorname{Liminf}_{n \rightarrow \infty} \frac{1}{\left|V_{n}\right|}\left(\varepsilon\left|V_{n}\right| \eta\right)=\varepsilon \eta,
$$

which is impossible. 
By Proposition 3, $K$ has finite right index $N$ in $\Gamma$, and we may write $\Gamma=\bigcup_{\tau \in T} K \tau$ where at most $N K \tau, \tau \in T$ have common intersection. Let

$$
T_{0}=T_{0}(n)=\left\{\tau \in T: K \tau \cap V_{n}^{\prime} \neq \varnothing\right\} .
$$

Hence

$$
V_{n}^{\prime} \subseteq \bigcup_{\tau \in T_{0}} K \tau
$$

Let $\gamma_{\tau} \in K \tau \cap V_{n}^{\prime}$ for $\tau \in T_{0}$, implying $\tau \in K^{-1} \gamma_{\tau}$ and $K \tau \subseteq K \cdot K^{-1} \gamma_{\tau}$, and therefore

$$
K V_{n}^{\prime} \subseteq \bigcup_{\tau \in T_{0}} K^{\prime} \gamma_{\imath}
$$

where each $\gamma_{\tau} \in V_{n}^{\prime}$. Let $M=\operatorname{Sup}_{y \in K} \Delta(\gamma)$. Since $\gamma_{\tau} \in K \tau$ for $\tau \in T_{0}$ we conclude

$$
\Delta\left(\gamma_{\tau}\right) \leqq M \Delta(\tau)
$$

Now assume $n_{0}$ is chosen so large that for $n \geqq n_{0}, \delta_{n}<|K| / M$. By the construction, $\gamma \in V_{n}^{\prime}$ implies $\left|K^{\prime} \sim V_{n} \gamma^{-1}\right| \leqq \delta_{n}$ and therefore $\left|K^{\prime} \gamma \sim V_{n}\right| \leqq \delta_{n} \Delta(\gamma)$. Also, if $V$ is a Borel set of $\Gamma$,

$$
\sum_{\tau \in T}|K \tau \cap V| \leqq N|V|
$$

by Fubini's Theorem, since any point of $\Gamma$ lies in at most $N$ different $K \tau, \tau \in T$. Since $e \in K$, for $\tau \in T_{0}$

$$
\left|K \tau \sim V_{n}\right| \leqq\left|K^{\prime} \gamma_{\tau} \sim V_{n}\right| \leqq \delta_{n} \Delta\left(\gamma_{\tau}\right) \leqq \delta_{n} M \Delta(\tau),
$$

and consequently

$$
\left|K \tau \cap V_{n}\right| \geqq|K \tau|-\delta_{n} M \Delta(\tau)=\left(|K|-\delta_{n} M\right) \Delta(\tau) .
$$

Hence by (2.6) and (2.8)

$$
N\left|V_{n}\right| \geqq \sum_{\tau \in T}\left|K \tau \cap V_{n}\right| \geqq \sum_{\tau \in T_{0}}\left|K \tau \cap V_{n}\right| \geqq\left(|K|-\delta_{n} M\right) \sum_{\tau \in T_{0}} \Delta(\tau),
$$

and therefore for $n \geqq n_{0}$

$$
\sum_{\tau \in T_{0}} \Delta(\tau) \leqq \frac{N\left|V_{n}\right|}{|K|-\delta_{n} M}
$$

But also

$$
K V_{n}^{\prime} \subseteq \bigcup_{\tau \in T_{0}} K^{\prime} \gamma_{\tau} \subseteq \bigcup_{\tau \in T_{0}}\left(K^{\prime} \gamma_{\tau} \sim V_{n}\right) \cup V_{n},
$$

and therefore by (2.7) and (2.9)

$$
\begin{aligned}
\left|K V_{n}^{\prime}\right| & \leqq \sum_{\tau \leq T_{0}}\left|K^{\prime} \gamma_{\tau} \sim V_{n}\right|+\left|V_{n}\right| \leqq \delta_{n} M \sum_{\tau \in T_{0}} \Delta(\tau)+\left|V_{n}\right| \\
& \leqq\left(1+\frac{\delta_{n} M N}{|K|-\delta_{n} M}\right)\left|V_{n}\right| .
\end{aligned}
$$


Finally, since $\delta_{n} \rightarrow 0$ as $n \rightarrow \infty$ and $\left|V_{n}^{\prime}\right| \geqq(1-\varepsilon)\left|V_{n}\right|$ for $n \in N^{+}$,

$$
\underset{n \rightarrow \infty}{\operatorname{Limsup}} \frac{\left|K V_{n}^{\prime}\right|}{\left|V_{n}^{\prime}\right|} \leqq \operatorname{Limsup}_{n \rightarrow \infty}\left(1+\frac{\delta_{n} M N}{|K|-\delta_{n} M}\right) \frac{\left|V_{n}\right|}{\left|V_{n}^{\prime}\right|}=\operatorname{Limsup}_{n \rightarrow \infty} \frac{\left|V_{n}\right|}{\left|V_{n}^{\prime}\right|} \leqq \frac{1}{1-\varepsilon} .
$$

TheOREM 15 (The APPROXimATION THEOREM). Let $\Gamma$ be any locally compact group, and let $\left\{V_{n}\right\} \in W_{\Gamma}$. Then there exists $\left\{V_{n}^{*}\right\} \in S_{\Gamma}^{0}$ such that $V_{n}^{*} \subseteq V_{n}$ for all $n \in N^{+}$and $\operatorname{Lim}_{n \rightarrow \infty}\left|V_{n} \sim V_{n}^{*}\right| /\left|V_{n}\right|=0$.

A sequence $\left\{V_{n}\right\}$ of Borel sets of $\Gamma$ with finite nonzero measure is in $W_{\Gamma}$ if and only if there exists $V_{n}^{*} \in S_{\Gamma}^{0}$ such that $\operatorname{Lim}_{n \rightarrow \infty}\left|V_{n} \triangle V_{n}^{*}\right| /\left|V_{n}\right|=0$.

Proof. By the regularity of Haar measure we may find Borel sets $V_{n}^{0} \subseteq V_{n}$ for all $n \in N^{+}$such that $\left\{V_{n}^{0}\right\} \in W_{\Gamma}^{0}$ and $\operatorname{Lim}_{n \rightarrow \infty}\left|V_{n} \sim V_{n}^{0}\right| /\left|V_{n}\right|=0$. Hence we may assume $\left\{V_{n}\right\} \in W_{\Gamma}^{0}$ initially. Furthermore, $\Gamma$ must be $\sigma$-compact if $W_{\Gamma} \neq \varnothing$ by Theorem 4, say $\Gamma=\bigcup C_{i}$ where all the countably many $C_{i}$ are compact. But this readily leads to the construction of a sequence $\left\{K_{i}\right\}$ of Borel sets of $\Gamma$ with compact closure, nonvoid interior, and all containing the identity such that

(1) $K_{i} \subseteq K_{i+1}, i \in N^{+}$.

(2) $\bigcup_{i}$ interior $\left(K_{i}\right)=\Gamma$.

(3) $K_{i}$ has finite right index in $\Gamma$.

For set $C_{i}^{\prime}=\bigcup_{j \leqq i} C_{j} \cup\{e\}$ and let $U$ be any open neighborhood of $e$ with compact closure. Then it is easily verified that the above conditions are satisfied by $K_{i}=U C_{i}^{\prime}$. Note that (3) follows directly from Proposition 3.

We now apply Lemma 4 to each $K=K_{i}$, taking $\varepsilon=\varepsilon_{i}=1 / 3 i$. Consequently there exists a sequence $\left\{V_{n, i}^{\prime}\right\}$ of Borel sets of $\Gamma$ satisfying

(i) $V_{n, i}^{\prime} \subseteq V_{n}$ for all $n \in N^{+}$.

(ii) $\left|V_{n} \sim V_{n, i}^{\prime}\right| \leqq(1 / 3 i)\left|V_{n}\right|$ for all $n \in N^{+}$.

(iii) Lim $\sup _{n \rightarrow \infty}\left|K_{\imath} V_{n, i}^{\prime}\right| /\left|V_{n, i}^{\prime}\right| \leqq 1 /(1-1 / 3 i)$.

From (iii) we infer the existence of $n_{0}=n_{0}(i)$ such that for all $n \geqq n_{0}$ we have

(iii)' $\left|K_{i} V_{n, i}^{\prime}\right| /\left|V_{n, i}^{\prime}\right|<1 /(1-1 / 2 i)$.

Note also that all $V_{n, i}^{\prime}$ have compact closure for sufficiently large $n$ by virtue of (i), since $\left\{V_{n}\right\} \in W_{\Gamma}^{0}$. We now construct $\left\{V_{n}^{*}\right\}$. Without loss of generality assume $n_{0}(i)$ is chosen such that $n_{0}(i)<n_{0}(i+1)$ for $i \in N^{+}$. For $1 \leqq n<n_{0}(1)$ let $V_{n}^{*}=V_{n}$, and for $i \in N^{+}$and $n_{0}(i) \leqq n<n_{0}(i+1)$ let $V_{n}^{*}=V_{n, i}^{\prime}$. Then each $V_{n}^{*} \subseteq V_{n}$ by (i), each $V_{n}^{*}$ is a Borel set of finite nonzero measure, and almost all $V_{n}^{*}$ have compact closure. Also by (ii), for $n_{0}(i) \leqq n<n_{0}(i+1),\left|V_{n} \sim V_{n}^{*}\right| /\left|V_{n}\right| \leqq 1 / 3 i$ and therefore $\operatorname{Lim}_{n \rightarrow \infty}\left|V_{n} \sim V_{n}^{*}\right| \mid$ $\left|V_{n}\right|=0$.

Finally to show that $\left\{V_{n}^{*}\right\} \in S_{\Gamma}^{0}$, let $C \neq \varnothing$ be a compact subset of $\Gamma$. Since $\cup$ interior $\left(K_{i}\right)=\Gamma$ is certainly an open cover of $C$ and the $K_{i}$ are nested, there exists an integer $i_{0}$ such that $C \subseteq K_{i}$ for $i \geqq i_{0}$. Therefore if $i \geqq i_{0}$ and $n_{0}(i) \leqq n<$ $n_{0}(i+1)$, we have by (iii)

$$
1 \leqq \frac{\left|C V_{n}^{*}\right|}{\left|V_{n}^{*}\right|} \leqq \frac{\left|K_{i} V_{n}^{*}\right|}{\left|V_{n}^{*}\right|}=\frac{\left|K_{i} V_{n, i}^{\prime}\right|}{\left|V_{n, i}^{\prime}\right|}<\frac{1}{1-1 / 2 i}
$$

and consequently $\operatorname{Lim}_{n \rightarrow \infty}\left|C V_{n}^{*}\right| /\left|V_{n}^{*}\right|=1$. 
We have just shown that if $\left\{V_{n}\right\} \in W_{\Gamma}$ there exists $\left\{V_{n}^{*}\right\} \in S_{\Gamma}^{0}$ such that

$$
\operatorname{Lim}_{n \rightarrow \infty}\left|V_{n} \triangle V_{n}^{*}\right| /\left|V_{n}\right|=0,
$$

and in fact $V_{n}^{*}$ could be taken to be a subset of $V_{n}$. Conversely, assume $\left\{V_{n}^{*}\right\} \in S_{\Gamma}^{0}$ and $\left\{V_{n}\right\}$ is a sequence of Borel sets of $\Gamma$ with finite nonzero measure satisfying (2.10).

Fix any $\gamma \in \Gamma$. Since $\left\{V_{n}^{*}\right\} \in S_{\Gamma}^{0} \subseteq W_{\Gamma}^{0}$, we have $\operatorname{Lim}_{n \rightarrow \infty}\left|\gamma V_{n}^{*} \triangle V_{n}^{*}\right| /\left|V_{n}^{*}\right|=0$. But

$$
\gamma V_{n} \triangle V_{n} \subseteq\left(\gamma V_{n} \triangle \gamma V_{n}^{*}\right) \cup\left(\gamma V_{n}^{*} \triangle V_{n}^{*}\right) \cup\left(V_{n}^{*} \triangle V_{n}\right),
$$

and consequently,

$$
\begin{aligned}
\operatorname{Limsup}_{n \rightarrow \infty} \frac{\left|\gamma V_{n} \triangle V_{n}\right|}{\left|V_{n}\right|} & \leqq \operatorname{Limsup}_{n \rightarrow \infty} \frac{\left|\gamma V_{n} \triangle \gamma V_{n}^{*}\right|+\left|\gamma V_{n}^{*} \triangle V_{n}^{*}\right|+\left|V_{n}^{*} \triangle V_{n}\right|}{\left|V_{n}\right|} \\
& =\operatorname{Limsup}_{n \rightarrow \infty} \frac{\left.2\left|V_{n} \triangle V_{n}^{*}\right|+\left|\gamma V_{n}^{*} \triangle V_{n}^{*}\right|\right)}{\left|V_{n}\right|}=0
\end{aligned}
$$

since $\operatorname{Lim}_{n \rightarrow \infty}\left|V_{n}\right| /\left|V_{n}^{*}\right|=1$; and therefore $\left\{V_{n}\right\} \in W_{\Gamma}$.

As an immediate corollary to The Approximation Theorem we have:

Corollary 6. $W_{\Gamma} \neq \varnothing \Leftrightarrow S_{\Gamma}^{0} \neq \varnothing$.

II.4. A necessary condition for $\left\{V_{n}\right\} \in W_{\Gamma}$. In this section we apply The Approximation Theorem to obtain a necessary condition for $\left\{V_{n}\right\} \in W_{\Gamma}$. It should be noted that the condition is also sufficient if $\Gamma$ is Abelian by Theorem 6. It is reasonable to expect that the condition stated in Theorem 16 is in fact sufficient in general also, but the author has been unable to prove this.

THEOREM 16. Let $\Gamma$ be a locally compact group, and let $\left\{V_{n}\right\} \in W_{\Gamma}$. Then for any Borel set $K$ of $\Gamma$ with compact closure and finite right index $N$ there exist subsets $T_{n} \subseteq \Gamma$ such that

$$
\operatorname{Lim}_{n \rightarrow \infty} \frac{\left|V_{n} \triangle \bigcup_{\tau \in T_{n}} K \tau\right|}{\left|V_{n}\right|}=0
$$

and at most $N$ of the $K \tau, \tau \in T_{n}$ intersect.

Proof. Since $\left\{V_{n}\right\} \in W_{\Gamma}$, by Theorem 15 there exists $\left\{V_{n}^{*}\right\} \in S_{\Gamma}^{0}$ such that $\operatorname{Lim}_{n \rightarrow \infty}\left|V_{n}^{*} \triangle V_{n}\right| /\left|V_{n}\right|=0$. Fix $K$ of right index $N$, say $\Gamma=\bigcup_{\tau \in T} K \tau$, where at most $N$ of the $K \tau, \tau \in T$ intersect. Let $K^{\prime}=K \cdot K^{-1}$ a Borel set of $\Gamma$ with compact closure. Since $\left\{V_{n}^{*}\right\} \in S_{\Gamma}^{0}, \operatorname{Lim}_{n \rightarrow \infty}\left|K^{\prime} V_{n}^{*}\right| /\left|V_{n}^{*}\right|=1$. Let $T_{n}=\left\{\tau \in T: K \tau \cap V_{n}^{*} \neq \varnothing\right\}$, implying

$$
V_{n}^{*} \subseteq \bigcup_{\tau \in T_{n}} K \tau
$$


Let $\alpha_{\tau} \in K \tau \cap V_{n}^{*}$ for $\tau \in T_{n}$, and hence $\tau \in K^{-1} \alpha_{\tau}$ and consequently $K \tau \subseteq K^{\prime} K^{-1} \alpha_{\tau}$ $=K^{\prime} \alpha_{\imath} \subseteq K^{\prime} V_{n}^{*}$. Therefore

Finally,

$$
\bigcup_{\tau \in T_{n}} K \tau \subseteq K^{\prime} V_{n}^{*}
$$

$$
\begin{aligned}
\underset{n \rightarrow \infty}{\operatorname{Limsup}} \frac{\left|V_{n} \triangle \bigcup_{\tau \in T_{n}} K \tau\right|}{\left|V_{n}\right|} & \leqq \operatorname{Limsup}_{n \rightarrow \infty}\left(\frac{\left|V_{n} \triangle V_{n}^{*}\right|+\left|\bigcup_{\tau \in T_{n}} K \tau \triangle V_{n}^{*}\right|}{\left|V_{n}\right|}\right) \\
& \leqq \operatorname{Limsup}_{n \rightarrow \infty}\left(\frac{\left|V_{n} \triangle V_{n}^{*}\right|+\left|K^{\prime} V_{n}^{*} \triangle V_{n}^{*}\right|}{\left|V_{n}\right|}\right)=0,
\end{aligned}
$$

and by construction at most $N$ of the $K \tau, \tau \in T_{n} \subseteq T$ intersect.

III. A generalization and some special considerations. In this section we use combinatorial arguments to study a generalization of the (weak) ratio property in the case where the locally compact group involved is a finitely generated discrete Abelian group. We first make a definition:

Definition 4. Let $\Lambda=\Gamma \times S$ be the product measure space of the locally compact topological group $(\Gamma, B,|\cdot|)$, where $B$ is taken to be the Borel sets of $\Gamma$ and $|\cdot|$ is a left invariant Haar measure on $B$, and the arbitrary measure space $(S, \Sigma,|\cdot|)$. A sequence $\left\{\Lambda_{n}\right\}$ of measurable subsets of $\Lambda$ with finite nonzero measure is said to satisfy the ratio property with respect to $\Gamma$ in $\Lambda$, written $\left\{\Lambda_{n}\right\} \in W_{\Gamma, \Lambda}$, if and only if $\operatorname{Lim}_{n \rightarrow \infty}\left|\gamma \Lambda_{n} \triangle \Lambda_{n}\right| /\left|\Lambda_{n}\right|=0$ for all $\gamma \in \Gamma$, where $\gamma \Lambda_{0}=\{(\gamma \tau, s):(\tau, s) \in$ $\left.\Lambda_{0} \subseteq \Gamma \times S\right\}$ for any $\Lambda_{0} \subseteq \Lambda=\Gamma \times S$. Note that if $S$ is a singleton of unit measure this definition collapses to the weak ratio property of Definition 1 .

If $a$ and $b$ are real numbers, we shall use the notation $a \wedge b=\operatorname{Min}\{a, b\}$, $a \vee b=\operatorname{Max}\{a, b\}$.

LEMMA 4. Let $b_{1}, \ldots, b_{r}$ be $r \geqq 2$ real numbers, and set

$$
S=\sum_{1 \leqq i \leqq r} b_{i}, \quad S_{0}=\frac{b_{1}+b_{r}}{2}+\sum_{1<i<r} b_{i}, \quad M=\sum_{1 \leqq i \leqq r} b_{i} \wedge b_{i+1} .
$$

Then

$$
S-M \geqq \frac{1}{r} S \geqq \frac{1}{r-1} M, \quad S_{0}-M \geqq \frac{1}{2} \operatorname{Max}_{1 \leqq i<j \leqq r}\left|b_{i}-b_{j}\right| .
$$

Proof. From the identity $a \wedge b=(a+b) / 2-|a-b| / 2$ we derive

$$
M=\sum_{1 \leqq i<r}\left(\frac{b_{i}+b_{i+1}}{2}-\frac{\left|b_{i}-b_{i+1}\right|}{2}\right)=S_{0}-\frac{1}{2} \sum_{1 \leqq i<r}\left|b_{i}-b_{i+1}\right| .
$$

And hence by the triangle inequality

$$
S_{0}-M \geqq \frac{1}{2} \operatorname{Max}_{1 \leqq i<j \leqq r}\left|b_{i}-b_{j}\right| .
$$

Also if we set $b=b_{m}=\operatorname{Max}_{1 \leq i \leq r} b_{i}$,

$$
M \leqq S_{0}-\frac{1}{2}\left(\left|b_{1}-b_{m}\right|+\left|b_{m}-b_{r}\right|\right)=S-b_{m} \leqq S-S / r,
$$

and consequently $S-M \geqq S / r, S / r \geqq M /(r-1)$. 
TheOREM 17. Let $\Lambda=Z \times S$ as in Definition 4 where $Z$ is the discrete group of integers with the counting measure. Let $\Lambda_{0}$ be any measurable subset of $\Lambda$ with finite nonzero measure and assume

$$
\left|\left(1+\Lambda_{0}\right) \cap \Lambda_{0}\right| \geqq(1-\varepsilon)\left|\Lambda_{0}\right| .
$$

Then if $c \in N^{+}$, we may partition $\Lambda_{0}$ as

$$
\Lambda_{0}=\left(\bigcup_{n} A_{n} \times S_{n}\right) \cup R
$$

where $n$ ranges over an appropriate index set $I=I(c)$, each $A_{n}$ consists of exactly $c$ consecutive integers, the $A_{n}$ are mutually disjoint, each $S_{n} \in \Sigma$, and $|R| \leqq 6 c \sqrt{ } \varepsilon\left|\Lambda_{0}\right|$.

Proof. The measurable sets of $\Lambda$ are precisely the sets of the form $\bigcup_{n \in Z}\{n\} \times B_{n}$ where $B_{n} \in \Sigma$ for $n \in Z$. Hence assume $\Lambda_{0}=\bigcup_{n \in Z}\{n\} \times B_{n}$ and let $b_{n}=\left|B_{n}\right|$. Then $\left|\Lambda_{0}\right|=\sum_{n \in Z} b_{n}$ and

$$
1+\Lambda_{0}=\bigcup_{n \in Z}\{n\} \times B_{n-1}, \quad\left(1+\Lambda_{0}\right) \cap \Lambda_{0}=\bigcup_{n \in Z}\left(\{n\} \times B_{n} \cap B_{n-1}\right) .
$$

By hypothesis,

$$
\begin{aligned}
\varepsilon\left|\Lambda_{0}\right| \geqq\left|\Lambda_{0} \sim\left(\left(1+\Lambda_{0}\right) \cap \Lambda_{0}\right)\right| & =\sum_{n \in \mathbf{Z}}\left(b_{n}-\left|B_{n} \cap B_{n-1}\right|\right) \\
& =\sum_{n \in Z}\left(b_{n-1}-\left|B_{n} \cap B_{n-1}\right|\right),
\end{aligned}
$$

and consequently

$$
2 \varepsilon\left|\Lambda_{0}\right| \geqq \sum_{n \in Z}\left(b_{n} \vee b_{n-1}-\left|B_{n} \cap B_{n-1}\right|\right) .
$$

Fix any $c \in N^{+}$and let $V_{n}=\{n\} \times B_{n} \cap B_{n-1}, A_{n}=\{c n, c n+1, \ldots, c n+c-1\}$, and $\Delta_{n}=\bigcup_{K \in A_{n}} V_{K}$. Therefore $\left(1+\Lambda_{0}\right) \cap \Lambda_{0}=\bigcup_{n \in Z} \Delta_{n}$ and

$$
\begin{aligned}
\varepsilon\left|\Lambda_{0}\right| & \geqq \sum_{n \in Z}\left(\sum_{K \in A_{n}}\left\{b_{K}-\left|B_{K} \cap B_{K-1}\right|\right\}\right) \\
& \geqq \sum_{n \in Z}\left(b_{c n}+\cdots+b_{c n+c-1}-\sum_{K \in A_{n}}\left(b_{K} \wedge b_{K-1}\right)\right) \\
& =\sum_{n \in Z}\left(\frac{b_{c n-1}}{2}+b_{c n}+\cdots+\frac{b_{c n+c-1}}{2}-\sum_{K \in A_{n}}\left(b_{K} \wedge b_{K-1}\right)\right) \\
& \geqq \frac{1}{2} \sum_{n \in Z} \operatorname{Max}\left\{\left|b_{u}-b_{v}\right|: u, v \in A_{n} \cup\{c n-1\}\right\}
\end{aligned}
$$

by Lemma 4. Now fix any $\delta, 0<\delta<1$, and let

$$
E=E_{\delta}=\left\{m \in Z:\left|B_{m} \cap B_{m-1}\right| \leqq(1-\delta)\left(b_{m} \vee b_{m-1}\right)\right\} .
$$

Then by (3.2)

$$
\begin{aligned}
2 \varepsilon\left|\Lambda_{0}\right| & \geqq \sum_{n \in \mathbf{Z}}\left(b_{n} \vee b_{n-1}-\left|B_{n} \cap B_{n-1}\right|\right) \geqq \sum_{m \in E}\left(b_{m} \vee b_{m-1}-\left|B_{m} \cap B_{m-1}\right|\right) \\
& \geqq \delta \sum_{m \in E} b_{m} \vee b_{m-1} \geqq \delta \sum_{m \in E} b_{m}
\end{aligned}
$$


Now let $E^{*}=\left\{n \in Z: A_{n} \cap E \neq \varnothing\right\}$. We shall show

$$
\sum_{n \in E^{*}}\left|\Delta_{n}\right| \leqq\left(\frac{2 c \varepsilon}{\delta}+2 c \varepsilon\right)\left|\Lambda_{0}\right|
$$

For, in general by Lemma 4,

$$
\left|\Delta_{n}\right| \leqq b_{c n-1} / 2+b_{c n}+\cdots+b_{c n+c-1} / 2
$$

and consequently

$$
\sum_{n \in E^{*}}\left|\Delta_{n}\right| \leqq \sum_{n \in E^{*}}\left\{c b_{m}+\left(\frac{1}{2}\left(b_{c n-1}-b_{m}\right)+\left(b_{c n}-b_{m}\right)+\cdots+\frac{1}{2}\left(b_{c n+c-1}-b_{m}\right)\right)\right\}
$$

where since $A_{n} \cap E \neq \varnothing$ we have taken any $m \in A_{n} \cap E$ and the corresponding term $b_{m}$. Hence,

$$
\begin{aligned}
\sum_{n \in E^{*}}\left|\Delta_{n}\right| & \leqq c \sum_{n \in E^{*}} b_{m}+\sum_{n \in E^{*}}\left\{\frac{1}{2}\left|b_{c n-1}-b_{m}\right|+\cdots+\frac{1}{2}\left|b_{c n+c-1}-b_{m}\right|\right\} \\
& \leqq c \sum_{m \in E} b_{m}+c \sum_{n \in E^{*}} \operatorname{Max}\left\{\left|b_{u}-b_{v}\right|: u, v \in\{c n-1, \ldots, c n+c-1\}\right\} \\
& \leqq \frac{2 c \varepsilon}{\delta}\left|\Lambda_{0}\right|+2 c \varepsilon\left|\Lambda_{0}\right|
\end{aligned}
$$

by (3.4) and (3.3).

We now set $I=Z \sim E^{*}$, and therefore $n \in I$ implies $A_{n} \cap E=\varnothing$, i.e. $\left|V_{K}\right|>$ $(1-\delta)\left(b_{K} \vee b_{K-1}\right)$ for all $K \in A_{n}$ where $V_{K}=\{K\} \times\left(B_{K} \cap B_{K-1}\right)$. For any $n \in I$, set

$$
S_{n}=\bigcap_{K \in\{c n-1, \ldots, c n+c-1\}} B_{K} \in \Sigma \text {. }
$$

We next prove for $n \in I$,

$$
\left|\Lambda_{n} \sim\left(A_{n} \times S_{n}\right)\right| \leqq c \delta \sum_{T \in A_{n}} b_{T} .
$$

For $k \in A_{n},\left|B_{k} \cap B_{k-1}\right|>(1-\delta)\left(b_{k} \vee b_{k-1}\right)$, and therefore $\left|B_{k} \sim B_{k-1}\right|<\delta b_{k}$, $\left|B_{k-1} \sim B_{k}\right|<\delta b_{k-1}$. But for any $k \in A_{n}$,

$$
\begin{aligned}
S_{n} & =\underset{T \in\{c n-1, \ldots, c n+c-1\}}{B_{T}} \\
& \supseteq\left(B_{k} \cap B_{k-1}\right) \sim\left\{\left(B_{k-1} \sim B_{k-2}\right) \vee \cdots \vee\left(B_{c n} \sim B_{c n-1}\right)\right. \\
\cup\left(B_{k} \sim B_{k+1}\right) & \left.\vee \cdots \vee\left(B_{c n+c-2} \sim B_{c n+c-1}\right)\right\},
\end{aligned}
$$

and therefore

$$
\left|S_{n}\right| \geqq\left|B_{k} \cap B_{k-1}\right|-\delta \sum_{T \in A_{n}} b_{T}
$$

Thus

$$
\left|\Lambda_{n} \sim\left(A_{n} \times S_{n}\right)\right|=\sum_{k \in A_{n}}\left(\left|B_{k} \cap B_{k-1}\right|-\left|S_{n}\right|\right) \leqq \sum_{k \in A_{n}} \delta \sum_{T \in A_{n}} b_{T}=c \delta \sum_{T \in A_{n}} b_{T}
$$

Consequently,

$$
\sum_{n \in I}\left|\Lambda_{n} \sim\left(A_{n} \times S_{n}\right)\right| \leqq c \delta \sum_{n \in I} \sum_{T \in A_{n}} b_{T} \leqq c \delta\left|\Lambda_{0}\right|
$$


and finally by (3.1), (3.5) and (3.7),

$$
\begin{aligned}
\left|\Lambda_{0} \sim \bigcup_{n \in I}\left(A_{n} \times S_{n}\right)\right| & =\left|\Lambda_{0} \sim\left(\Lambda_{0} \cap\left(1+\Lambda_{0}\right)\right)\right|+\left|\left(\Lambda_{0} \cap\left(1+\Lambda_{0}\right)\right) \sim \bigcup_{n \in I}\left(A_{n} \times S_{n}\right)\right| \\
& =\left|\Lambda_{0} \sim\left(\Lambda_{0} \cap\left(1+\Lambda_{0}\right)\right)\right|+\sum_{n \in E^{*}}\left|\Delta_{n}\right|+\sum_{n \in I}\left|\Delta_{n} \sim\left(A_{n} \times S_{n}\right)\right| \\
& \leqq \varepsilon\left|\Lambda_{0}\right|+\left(\frac{2 c \varepsilon}{\delta}\left|\Lambda_{0}\right|+2 c \varepsilon\left|\Lambda_{0}\right|\right)+c \delta\left|\Lambda_{0}\right| .
\end{aligned}
$$

Upon setting $\delta=\sqrt{ } \varepsilon$, we obtain the theorem.

COROllary 7. There exist two functions $\omega_{1}$ and $\delta_{1}$ defined on $(0,1)$ satisfying $\operatorname{Lim}_{\varepsilon \rightarrow 0^{+}} \delta_{1}(\varepsilon)=0, \quad \operatorname{Lim}_{\varepsilon \rightarrow 0^{+}} \omega_{1}(\varepsilon)=+\infty$ such that $\left|\Lambda_{0} \cap\left(1+\Lambda_{0}\right)\right| \geqq(1-\varepsilon)\left|\Lambda_{0}\right|$ implies

$$
\Lambda_{0}=\left(\bigcup_{n} A_{n} \times S_{n}\right) \cup R
$$

where each $A_{n}$ consists of exactly $\omega_{1}(\varepsilon)$ consecutive integers, the $A_{n}$ are pairwise disjoint, $S_{n} \in \Sigma$, and $|R| \leqq \delta_{1}(\varepsilon)\left|\Lambda_{0}\right|$. In fact, we may take $\omega_{1}(\varepsilon)=\left[\varepsilon^{-1 / 4}\right], \delta_{1}(\varepsilon)$ $=6 \varepsilon^{1 / 4}$.

Proof. Set $c=\left[\varepsilon^{-1 / 4}\right]$ in Theorem 17 , and note that we may take $\delta_{1}(\varepsilon)$ $=6 \sqrt{ } \varepsilon\left[\varepsilon^{-1 / 4}\right] \leqq 6 \varepsilon^{1 / 4}$.

We now generalize Corollary 7 :

THEOREM 18. Let $\Lambda=Z^{k} \times S$ be the product measure space of $Z^{k}\left(k \in N^{+}\right)$with the counting measure and the measure space $S$. Let $e_{j}=\left(\delta_{i j}, \ldots, \delta_{k j}\right) \in Z^{k}$ for $1 \leqq j \leqq k$. Then there exist two functions $\omega_{k}$ and $\delta_{k}$ defined on $(0,1)$ satisfying $\operatorname{Lim}_{\varepsilon \rightarrow 0^{+}} \delta_{k}(\varepsilon)=0, \operatorname{Lim}_{\varepsilon \rightarrow 0^{+}} \omega_{k}(\varepsilon)=+\infty$ such that

$$
\left|\Lambda_{0} \cap\left(e_{j}+\Lambda_{0}\right)\right| \geqq(1-\varepsilon)\left|\Lambda_{0}\right| \text { for all } j, 1 \leqq j \leqq k,
$$

implies

$$
\Lambda_{0}=\left(\bigcup_{n} A_{1}^{n} \times \cdots \times A_{k}^{n} \times S_{n}\right) \cup R^{\prime}
$$

where $\left|R^{\prime}\right| \leqq \delta_{k}(\varepsilon)\left|\Lambda_{0}\right|$, each $A_{i}^{n}$ is a set of at least $\omega_{k}(\varepsilon)$ consecutive integers, $A^{n}$ $=A_{1}^{n} \times \cdots \times A_{k}^{n}$ are pairwise disjoint, and $S_{n} \in \Sigma$. In fact, we may take

$$
\omega_{k}(\varepsilon)=\left[\varepsilon^{-\lambda_{k}}\right], \quad \delta_{k}(\varepsilon)=\left(7 k^{2}+7 k-2\right) \varepsilon^{\wedge_{k}} / 2,
$$

where

$$
\lambda_{k}=\left\{\left(\frac{2+\sqrt{ } 2}{4}\right)(2+2 \sqrt{ } 2)^{k}+\left(\frac{2-\sqrt{ } 2}{4}\right)(2-2 \sqrt{ } 2)^{k}\right\}^{-1} .
$$

Proof. We proceed by induction, noting that Corollary 7 covers the case $k=1$. Assume the theorem true for $j<k$. Let $\Lambda=Z^{k} \times S=Z \times S^{*}$ where $S^{*}=Z^{k-1} \times S$. We shall find it convenient to consider $\Lambda$ decomposed as a product in both these ways. Assume $\Lambda_{0} \subseteq \Lambda$ is such that $\left|\Lambda_{0} \cap\left(e_{j}+\Lambda_{0}\right)\right| \geqq\left(1-\varepsilon^{\rho}\right)\left|\Lambda_{0}\right|$ for $1 \leqq j \leqq k$, where $\rho>4$ is a parameter to be optimally chosen later. Then in particular considering 
$\Lambda=Z \times S^{*}$, in which case translation by $e_{1}$ corresponds to translating $Z$ by 1 , we obtain from Corollary 7

$$
\Lambda_{0}=\left(\bigcup A_{n} \times S_{n}^{*}\right) \cup R,
$$

where $|R| \leqq 6 \varepsilon^{\rho / 4}\left|\Lambda_{0}\right|,\left|A_{n}\right|=\left[\varepsilon^{-\rho / 4}\right]$, the $A_{n}$ are pairwise disjoint, $S_{n}^{*} \subseteq S^{*}$ and measurable. For $2 \leqq j \leqq k,\left|\Lambda_{0} \cap\left(e_{j}+\Lambda_{0}\right)\right| \geqq\left(1-\varepsilon^{\rho}\right)\left|\Lambda_{0}\right|$; let $I_{j}$ index all $n$ in the above representation for which $\left|S_{n}^{*} \cap\left(e_{j}+S_{n}^{*}\right)\right| \leqq(1-\varepsilon)\left|S_{n}^{*}\right|(2 \leqq j \leqq k)$, where we interpret $e_{j}+S_{n}^{*}$ by projecting $e_{j}$ on its last $(k-1)$ factors and consider $S_{n}^{*} \subseteq Z^{k-1} \times S$ in the natural way. We then have for $2 \leqq j \leqq k$

$\varepsilon^{\rho}\left|\Lambda_{0}\right| \geqq\left|\Lambda_{0} \sim\left\{\left(e_{j}+\Lambda_{0}\right) \cap \Lambda_{0}\right\}\right| \geqq\left|\bigcup_{n} A_{n} \times\left\{S_{n}^{*} \sim\left(S_{n}^{*} \cap\left(e_{j}+S_{n}^{*}\right)\right)\right\}\right|-6 \varepsilon^{\rho / 4}\left|\Lambda_{0}\right|$, implying

$$
7 \varepsilon^{0 / 4}\left|\Lambda_{0}\right| \geqq \varepsilon \sum_{n \in I_{j}}\left|A_{n} \times S_{n}^{*}\right|
$$

Hence

$$
\Lambda_{0}=\left(\bigcup_{n}^{\prime} A_{n} \times S_{n}^{*}\right) \cup R^{*}
$$

where

$R^{*}=\left(\bigcup_{n \in I_{2} \cup \cdots \cup I_{k}} A_{n} \times S_{n}^{*}\right) \cup R, \quad\left|R^{*}\right| \leqq\left(6 \varepsilon^{\rho / 4}+7 \varepsilon^{\rho / 4-1}(k-1)\right)\left|\Lambda_{0}\right| \leqq 7 k \varepsilon^{0 / 4-1}\left|\Lambda_{0}\right|$,

and the restricted union $U^{\prime}$ is over $n \notin I_{2} \cup \cdots \cup I_{k}$. By construction, for every such $n$,

$$
\left|S_{n}^{*} \cap\left(e_{j}+S_{n}^{*}\right)\right| \geqq(1-\varepsilon)\left|S_{n}^{*}\right| \text { for } 2 \leqq j \leqq k,
$$

and by applying the induction hypothesis to $S_{n}^{*}$,

$$
S_{n}^{*}=\left(\bigcup_{i} A_{2, i}^{n} \times \cdots \times A_{k, i}^{n} \times S_{n, i}\right) \cup R_{n}
$$

where $\left|R_{n}\right| \leqq \delta_{k-1}(\varepsilon)\left|S_{n}^{*}\right|$, each $A_{j, i}^{n}$ is a set of at least $\omega_{k-1}(\varepsilon)$ consecutive integers, and the $A_{2, i}^{n} \times \cdots \times A_{k, i}^{n}$ are disjoint. Therefore for such $n$

$$
A_{n} \times S_{n}^{*}=\left(\bigcup_{i} A_{n} \times A_{2, i}^{n} \times \cdots \times A_{k, i}^{n} \times S_{n, i}\right) \cup \bar{R}_{n}
$$

where

$$
\bar{R}_{n}=A_{n} \times R_{n}, \quad\left|\bar{R}_{n}\right| \leqq \delta_{k-1}(\varepsilon)\left|A_{n} \times S_{n}^{*}\right| .
$$

Thus we finally obtain

$$
\Lambda_{0}=\left\{\bigcup_{n}^{\prime} \bigcup_{i}\left(A_{n} \times A_{2, i}^{n} \times \cdots \times A_{k, i}^{n} \times S_{n, i}\right)\right\} \cup R^{\prime}
$$

where $R^{\prime}=\bigcup_{n}^{\prime} \bar{R}_{n} \cup R^{*}$ and $\left|R^{\prime}\right| \leqq\left(\delta_{k-1}(\varepsilon)+7 \varepsilon^{\rho / 4-1} k\right)\left|\Lambda_{0}\right|$. The double union is of terms of the desired type where each factor from a component of $Z^{k}$ consists of at least $\operatorname{Min}\left(\left[\varepsilon^{-\rho / 4}\right], \omega_{k-1}(\varepsilon)\right)$ consecutive integers. Hence we may take

$$
\delta_{k}(\varepsilon)=\delta_{k-1}\left(\varepsilon^{1 / \rho}\right)+7 e^{1 / 4-1 / 0} k, \quad \omega_{k}(\varepsilon)=\operatorname{Min}\left(\left[e^{-1 / 4}\right], \omega_{k-1}\left(e^{1 / \rho}\right)\right) .
$$


We now wish to choose $\rho_{0}=\rho_{0}(k)$ so as to optimize the estimate of $\delta_{k}$ inductively. Assume $\delta_{k-1}$ is of the form: $\delta_{k-1}(\varepsilon)=C_{k-1} \varepsilon^{\lambda_{k-1}}$. Then $\delta_{k}(\varepsilon)=C_{k-1} \varepsilon^{\lambda_{k-1 / \rho}}+$ $7 k \varepsilon^{1 / 4-1 / \rho}$ is admissible for any $\rho>4$. We choose $\rho_{0}$ such that $\lambda_{k-1} / \rho_{0}=1 / 4-1 / \rho_{0}$ and set $\lambda_{k}=\lambda_{k-1} / \rho_{0}$. In this way, if we set $\mu_{k}=1 / \lambda_{k}$ we obtain the recursions:

$$
\begin{aligned}
& \mu_{k}=4 \mu_{k-1}+4, \quad \mu_{1}=4, \\
& C_{k}=7 k+C_{k-1}, \quad C_{1}=6 .
\end{aligned}
$$

Consequently, $C_{k}=\left(7 k^{2}+7 k-2\right) / 2$ for $k \geqq 1$ and

$$
\mu_{k}=\left(\frac{2+\sqrt{ } 2}{4}\right)(2+2 \sqrt{ } 2)^{k}+\left(\frac{2-\sqrt{ } 2}{4}\right)(2-2 \sqrt{ } 2)^{k} \quad \text { for } k \geqq 1,
$$

by standard methods of linear difference equations.

The following simple lemma is used in proving the next theorem:

LEMMA 5. $\left\{\Lambda_{n}\right\} \in W_{\Gamma, \Lambda}$ if and only if $\operatorname{Lim}_{n \rightarrow \infty}\left|g \Lambda_{n} \triangle \Lambda_{n}\right| /\left|\Lambda_{n}\right|=0$ for all $g$ in $a$ generating set $G$ of $\Gamma$.

Proof. Let $\gamma \in \Gamma$, say $\gamma=g_{r} g_{r-1} \cdots g_{2} g_{1}, g_{i} \in G, 1 \leqq i \leqq r$, and choose $n_{0}(\varepsilon)$ such that for $n \geqq n_{0},\left|g_{i} \Lambda_{n} \triangle \Lambda_{n}\right| \leqq \varepsilon\left|\Lambda_{n}\right|$ for $1 \leqq i \leqq r$. Then for $n \geqq n_{0}$

$$
\begin{aligned}
\left|\gamma \Lambda_{n} \triangle \Lambda_{n}\right|= & \left|g_{r} \cdots g_{2} g_{1} \Lambda_{n} \triangle \Lambda_{n}\right| \leqq\left|g_{r} \cdots g_{1} \Lambda_{n} \triangle g_{r} \cdots g_{2} \Lambda_{n}\right| \\
& +\left|g_{r} \cdots g_{2} \Lambda_{n} \triangle g_{r} \cdots g_{3} \Lambda_{n}\right|+\cdots+\left|g_{r} \Lambda_{n} \triangle \Lambda_{n}\right| \\
= & \left|g_{1} \Lambda_{n} \triangle \Lambda_{n}\right|+\left|g_{2} \Lambda_{n} \triangle \Lambda_{n}\right|+\cdots+\left|g_{r} \Lambda_{n} \triangle \Lambda_{n}\right| \leqq r \varepsilon\left|\Lambda_{n}\right|
\end{aligned}
$$

and therefore, since $\varepsilon$ may be arbitrarily small, $\operatorname{Lim}_{n \rightarrow \infty}\left|\gamma \Lambda_{n} \triangle \Lambda_{n}\right| /\left|\Lambda_{n}\right|=0$.

THEOREM 19. Let $\Gamma=C \times Z^{k},|C|<+\infty$, be a finitely generated discrete Abelian group, $\Lambda=\Gamma \times S$ as in Definition 4 , and $\left\{\Lambda_{n}\right\}$ a sequence of measurable subsets of $\Lambda$ with finite nonzero measure. Then $\left\{\Lambda_{n}\right\} \in W_{\Gamma, \Lambda}$ if and only if there exist two sequences $\left\{\omega_{n}\right\},\left\{\gamma_{n}\right\}, \operatorname{Lim}_{n \rightarrow \infty} \omega_{n}=+\infty, \operatorname{Lim}_{n \rightarrow \infty} \gamma_{n}=0$, such that

$$
\Lambda_{n}=\left(\bigcup_{m} C \times A_{1}^{m} \times \cdots \times A_{k}^{m} \times S_{m}\right) \cup R_{n}
$$

where $\left|R_{n}\right| \leqq \delta_{n}\left|\Lambda_{n}\right|$, each $A_{i}^{m}$ is a set of at least $\omega_{n}$ consecutive integers, the $A_{i}^{m} \times \cdots$ $\times A_{k}^{m}$ are pairwise disjoint and each $S_{m} \in \Sigma$.

Proof. By Lemma 5 we need only verify the ratio property for a generating set for $\Gamma$; we take $G=C \cup\left\{e_{1}, \ldots, e_{k}\right\}$. For $c \in C, c+\Lambda_{n}=\left(\bigcup_{m}(c+C) \times A_{1}^{m} \times \cdots\right.$ $\left.\times A_{k}^{m} \times S_{m}\right) \cup\left(c+R_{n}\right)=\left(\bigcup_{m} C \times A_{1}^{m} \times \cdots \times A_{k}^{m} \times S_{m}\right) \cup R_{n}^{\prime}$ where $\left|R_{n}^{\prime}\right|=\left|R_{n}\right| \leqq \delta_{n}\left|\Lambda_{n}\right|$ and therefore $\left|\left(c+\Lambda_{n}\right) \cap \Lambda_{n}\right| \geqq\left(1-\delta_{n}\right)\left|\Lambda_{n}\right|$ implying $\operatorname{Lim}_{n \rightarrow \infty}\left|\left(c+\Lambda_{n}\right) \triangle \Lambda_{n}\right| /\left|\Lambda_{n}\right|$ $=0$. Similarly for $1 \leqq i \leqq k$,

$$
e_{i}+\Lambda_{n}=\left\{\bigcup_{m} C \times A_{1}^{m} \times \cdots \times\left(A_{i}^{m}+1\right) \times \cdots \times A_{k}^{m} \times S_{m}\right\} \cup R_{n}^{\prime}
$$


where $\left|R_{n}^{\prime}\right|=\left|R_{n}+e_{i}\right| \leqq \delta_{n}\left|\Lambda_{n}\right|$. Since $A_{i}^{m}$ consists of at least $\omega_{n}$ consecutive integers,

$$
\begin{aligned}
\left|\left(e_{i}+\Lambda_{n}\right) \cap \Lambda_{n}\right| & \geqq\left|\bigcup_{m} C \times A_{1}^{m} \times \cdots \times\left(A_{i}^{m} \cap\left(A_{i}^{m}+1\right)\right) \times \cdots \times A_{k}^{m} \times S_{m}\right| \\
& \geqq\left(1-\frac{1}{\omega_{n}}\right)\left|\bigcup_{m} C \times A_{1}^{m} \times \cdots \times A_{k}^{m} \times S_{m}\right| \geqq\left(1-\frac{1}{\omega_{n}}\right)\left(1-\delta_{n}\right)\left|\Lambda_{n}\right|,
\end{aligned}
$$

and hence $\operatorname{Lim}_{n \rightarrow \infty}\left|\left(e_{i}+\Lambda_{n}\right) \triangle \Lambda_{n}\right| /\left|\Lambda_{n}\right|=0$ since $\omega_{n} \rightarrow \infty, \delta_{n} \rightarrow 0$.

Conversely, let $C=\left\{c_{1}, \ldots, c_{T}\right\}$ and write

$$
\Lambda_{n}=\bigcup_{1 \leqq i \leqq T}\left\{c_{i}\right\} \times A_{n}^{i}
$$

where $A_{n}^{i} \subseteq Z^{k} \times S$. Choose $n_{0}=n_{0}(\varepsilon)$ such that for $n \geqq n_{0}, 1 \leqq i \leqq T$,

$$
\left|\left(C_{i}+\Lambda_{n}\right) \cap \Lambda_{n}\right| \geqq(1-\varepsilon)\left|\Lambda_{n}\right| \text {. }
$$

Therefore, for $1 \leqq i, j \leqq T,\left|A_{n}^{i} \sim A_{n}^{j}\right| \leqq \varepsilon\left|\Lambda_{n}\right|$. Let $\left|A_{n}^{i_{0}}\right|=\operatorname{Max}_{1 \leqq i \leqq T}\left|A_{n}^{i}\right|$, and

$$
A_{n}=\bigcap_{1 \leqq i \leqq T} A_{n}^{i}=\left\{\left\{\cdots\left\{A_{n}^{i_{0}} \sim\left(A_{n}^{i_{0}} \sim A_{n}^{i_{1}}\right)\right\} \sim \cdots\right\} \sim\left(A_{n}^{i_{T}-2} \sim A_{n}^{i_{T}-1}\right)\right\},
$$

where $i_{0}, i_{1}, \ldots, i_{T-1}$ is an ordering of $1,2, \ldots, T$. We then have

$$
\left|A_{n}\right| \geqq\left|A^{i_{0}}\right|-T \varepsilon\left|\Lambda_{n}\right| \geqq\left(\frac{1}{T}-T \varepsilon\right)\left|\Lambda_{n}\right|,
$$

and consequently $\Lambda_{n}=\left(C \times A_{n}\right) \cup R_{n}$ where $\left|R_{n}\right| \leqq T^{2} \varepsilon\left|\Lambda_{n}\right|$. Choose $n_{0}^{\prime}=n_{0}^{\prime}(\varepsilon)$ such that $n \geqq n_{0}^{\prime}$ implies $\left|\left(e_{i}+\Lambda_{n}\right) \cap \Lambda_{n}\right| \geqq(1-\varepsilon)\left|\Lambda_{n}\right|$ for $1 \leqq i \leqq k$. Then

$$
\begin{aligned}
\left|\left(e_{i}+\Lambda_{n}\right) \cap \Lambda_{n}\right| & =\left|\left\{\left(C \times\left(e_{i}+A_{n}\right)\right) \cup\left(e_{i}+R_{n}\right)\right\} \cap\left\{\left(C \times\left(A_{n} \cup R_{n}\right)\right)\right\}\right| \\
& \leqq\left|C \times\left(A_{n} \cap\left(e_{i}+A_{n}\right)\right)\right|+2\left|R_{n}\right|=T\left|A_{n} \cap\left(e_{i}+A_{n}\right)\right|+2\left|R_{n}\right| .
\end{aligned}
$$

Therefore for $1 \leqq i \leqq k$

$$
\begin{aligned}
\left|\left(e_{i}+A_{n}\right) \cap A_{n}\right| & \geqq \frac{(1-\varepsilon)\left|\Lambda_{n}\right|-2\left|R_{n}\right|}{T} \geqq\left(1-\varepsilon-2 T^{2} \varepsilon\right) \frac{\left|\Lambda_{n}\right|}{T} \\
& \geqq(1-(2 T+1) \varepsilon)\left|A_{n}\right| .
\end{aligned}
$$

The proof is now completed by applying Theorem 18 to $\Lambda_{0}=A_{n}$.

\section{BIBLIOGRAPHY}

1. P. R. Halmos, Measure theory, Van Nostrand, Princeton, N. J., 1961.

2. W. Emerson and F. P. Greenleaf, Covering properties and Folner conditions for locally compact groups, Math. Z. 102 (1967), 370-384.

\section{University of California,}

Berkeley, California 